University of Louisville

ThinkIR: The University of Louisville's Institutional Repository

Electronic Theses and Dissertations

$5-2010$

\title{
Computational fluid dynamic analysis of a coulter counting microcytometer.
}

Kyle Anthony Schmidt

University of Louisville

Follow this and additional works at: https://ir.library.louisville.edu/etd

\section{Recommended Citation}

Schmidt, Kyle Anthony, "Computational fluid dynamic analysis of a coulter counting microcytometer." (2010). Electronic Theses and Dissertations. Paper 1274.

https://doi.org/10.18297/etd/1274

This Master's Thesis is brought to you for free and open access by ThinkIR: The University of Louisville's Institutional Repository. It has been accepted for inclusion in Electronic Theses and Dissertations by an authorized administrator of ThinkIR: The University of Louisville's Institutional Repository. This title appears here courtesy of the author, who has retained all other copyrights. For more information, please contact thinkir@louisville.edu. 


\title{
COMPUTATIONAL FLUID DYNAMIC ANALYSIS OF A COULTER COUNTING MICROCYTOMETER
}

\author{
By \\ Kyle Anthony Schmidt \\ B.S, University of Louisville, 2009

\begin{abstract}
A Thesis
Submitted to the Faculty of the

University of Louisville

J.B. Speed School of Engineering

in Partial Fulfillment of the Requirements

for the Professional Degree
\end{abstract} \\ MASTER OF ENGINEERING \\ Department of Mechanical Engineering
}

May 2010 

COMPUTATIONAL FLUID DYNAMIC ANALYSIS OF A COULTER COUNTING MICROCYTOMETER

\author{
Submitted by: \\ Kyle Anthony Schmidt \\ A Thesis Approved On \\ (Date)
}

by the Following Reading and Examination Committee:

Yongsheng Lian, Thesis Director

Cindy K. Harnett

Michael K. Sharp 


\section{ACKNOLEDGMENTS}

To Dr. Harnett, whose work on microchannel cytometry would not have led to the production of this thesis.

To Bill Henshaw, for the use of Overture, without it no simulations would have been produced. His knowledge of the program gave way to solving many problems.

To Dr. Lian, for taking time to be my advisor and having enough patients to guide me through this process.

Lastly to everybody around me who had to listen, even though they did not want to, about the never ending headaches and solutions that came from doing this research and thesis. 


\begin{abstract}
This thesis looks at the optimization of fluid flow and mass transfer using computational fluid dynamics (CFD) in a micro fluidic coulter counter. CFD will be used to solve equations of fluid flow and mass transfer to see how molecules within the microchannel will react with different flow ratios. Choosing a sheath stream to sample stream flow ratio will optimize the channel by increasing the concentration level of the sample stream near the electrodes; resulting in a clearer and more precise signal. Optimization will provide cell separation between each passing cell to improve counting efficiency.

Analysis of the microcytometer shows the numerical solution of the fluid flow is greatly affected by a changing the $\mathrm{x}$ directional grid lines while having grid lines stretched in the y direction towards the microcytometer walls. The numerical diffusion solution is greatly affected by increasing or decreasing the y directional grid lines. While a sheath stream to sample stream flow ratio between 6.643 and 5.056 proved to have the highest sample stream concentration level and a focused stream width the same width as the cell diameter. Also a three dimensional numerical solution shows viscous effects of the fluid on the wall. This is represented by an hour glass shape of the diffusion.
\end{abstract}




\section{TABLE OF CONTENTS}

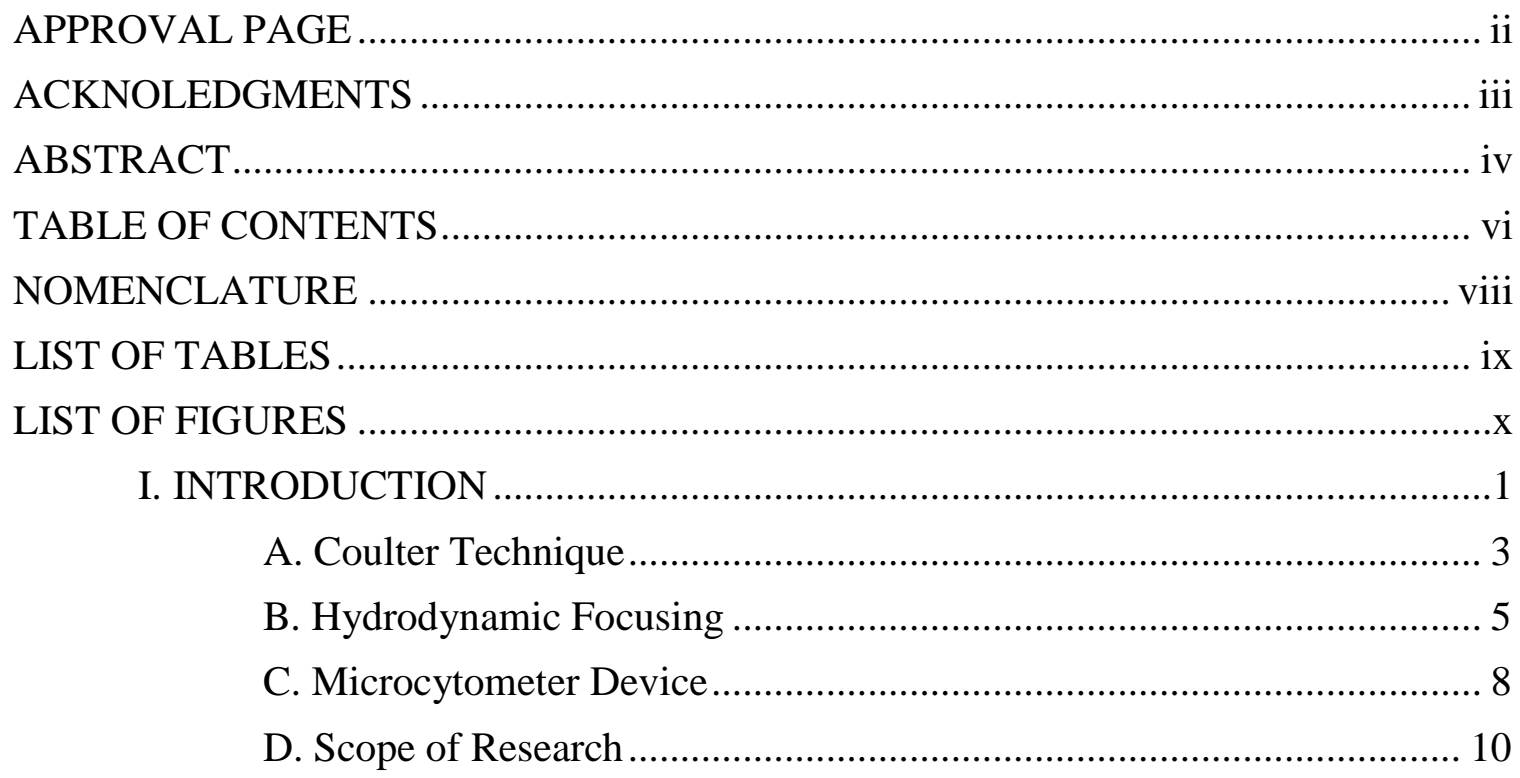

II. FLUID FLOW IN MICROCYTOMETERS USING NUMERICAL

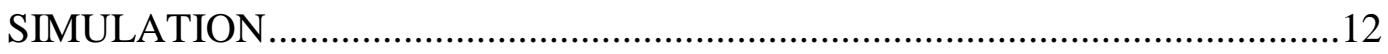

A. Ogen and Grid Generation ....................................................... 15

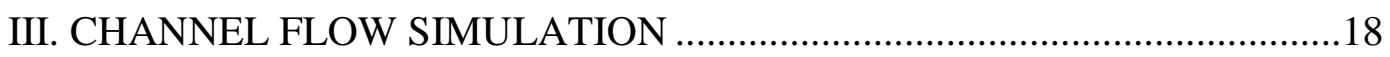

A. Flow Through A Single Channel ..................................................... 18

B. Microcytometer Grid: A Three Inlet Channel.................................. 26

C. Cell Motion in the Microcytometer................................................. 31

IV. MASS DIFFUSION WITHIN A MICROCYTOMETER ............................35

A. Experimental Diffusion................................................................ 38

B. Diffusion Grid Sensitivity ............................................................. 39

C. Effects of Different Asymmetric and Symmetrical Flow Rates ......... 44

V. THREE DIMENSIONAL MICROCYTOMETER .......................................50

A. Three Dimensional Fluid Flow and Diffusion ................................. 50

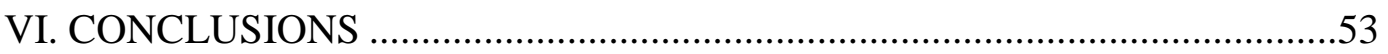




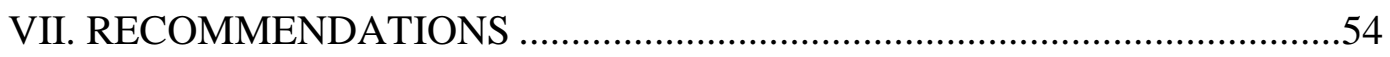

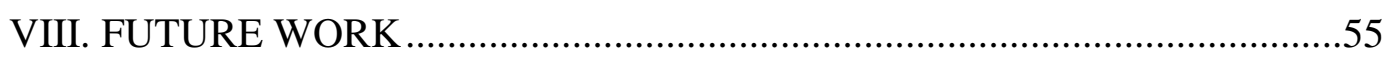

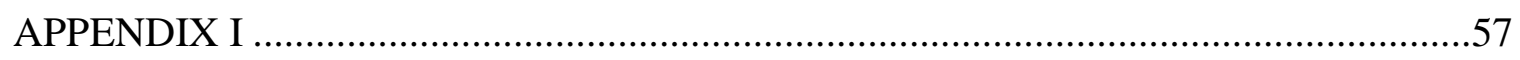

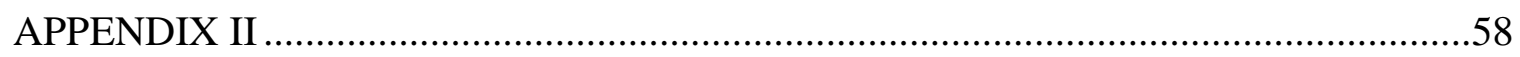

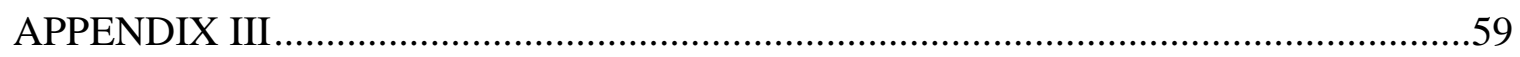

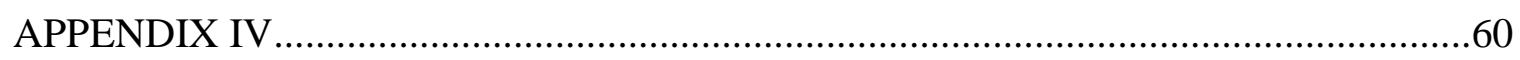

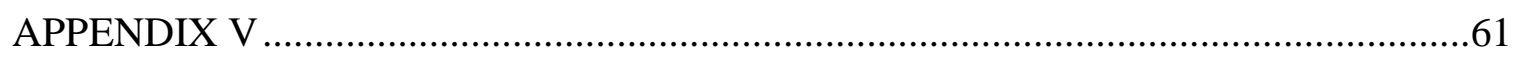

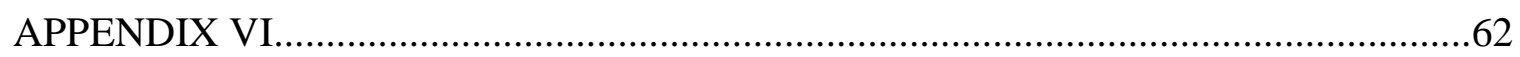

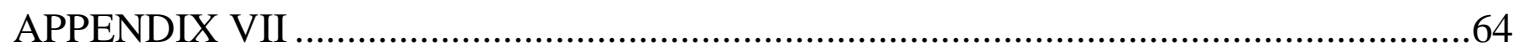

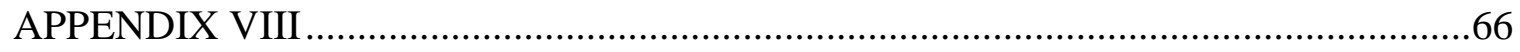

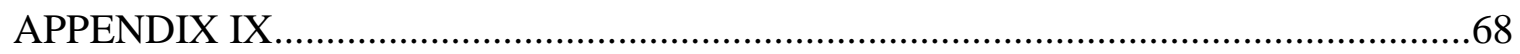

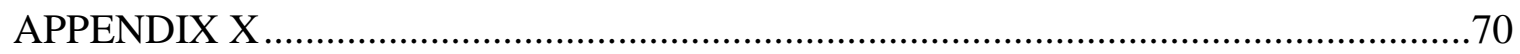

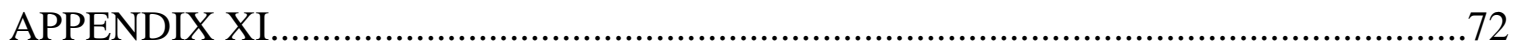

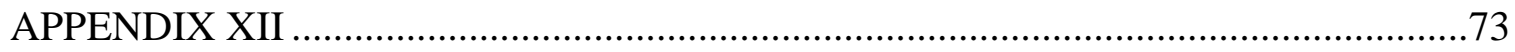

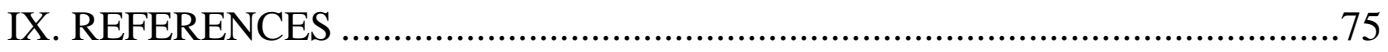

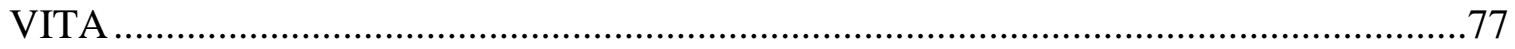




\section{NOMENCLATURE}

$\begin{aligned} & \mathrm{V}=\text { velocity } \\ & \mu=\text { } \text {-direction velocity } \\ & \mathrm{h}=\text { half the channel width } \\ & \mathrm{y}=\text { channel position } \\ & \mathrm{Re}=\text { Reynolds number } \\ & \rho=\text { density } \\ & \mathrm{d}_{\mathrm{h}}=\text { hydraulic dyameter } \\ & v=\text { kinematic viscosity } \\ & \mathrm{le}=\text { entrance length } \\ & \mathrm{P}=\text { pressure } \\ & \mathrm{N}=\text { Molar flow } \\ & \mathrm{A}=\text { cross section area } \\ & \mathrm{N} / \mathrm{A}=\text { Molar flux } \\ & \mathrm{D}=\text { mass diffusivity } \\ & \mathrm{Sc}=\text { Schmidt number } \\ & \mathrm{c}=\text { concentration } \\ & \mathrm{Pe}=\text { Peclet number } \\ & \mathrm{W}=\text { width of the channel } \\ & \mathrm{U}=\text { uniform velocity } \\ & \mathrm{Q} 1=\text { sample stream flow } \\ & \mathrm{Q} 2=\text { upper sheath flow } \\ & \mathrm{Q} 3=\text { lower sheath flow } \\ & \mathrm{W}=\text { width of the focused stream } \\ & \mathrm{D}_{\mathrm{m}}=\text { width of the microchannel } \\ & \alpha=\text { diffusion coefficient } \\ &\end{aligned}$




\section{LIST OF TABLES}

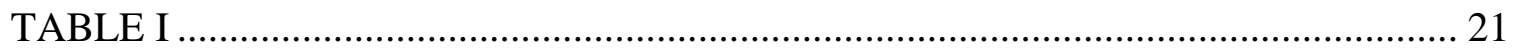

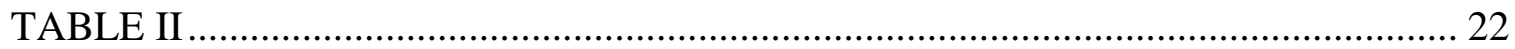

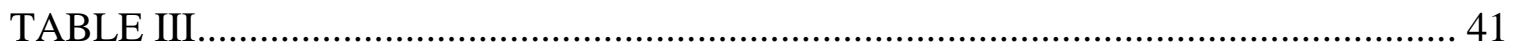

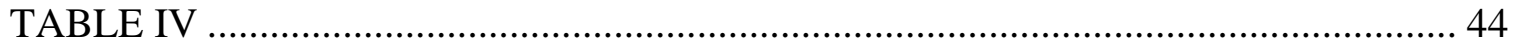

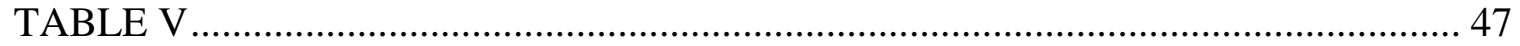




\section{LIST OF FIGURES}

FIGURE 1- Flow process in a cytometer............................................................... 2

FIGURE 2 - Process of the Coulter Technique. ............................................................. 4

FIGURE 3 - Experimental signal produced when a cell passes the electrodes.................. 5

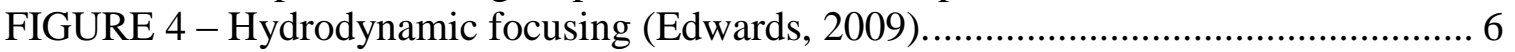

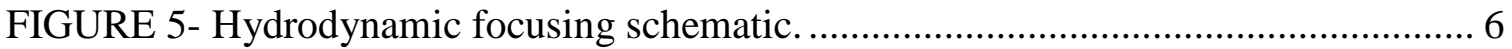

FIGURE 6 - Symmetric hydrodynamic focusing. ..................................................... 7

FIGURE 7 - Schematic of multiple domains occurring in microcytometer. ................... 10

FIGURE 8 - A simple overlapping grid. Left: the overlapping grid, right: overlapping

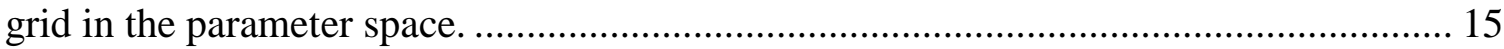

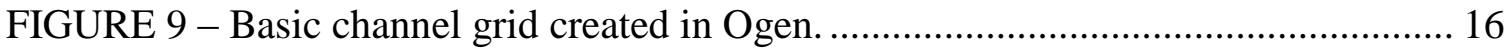

FIGURE 10 - Velocity profile comparison between the basic numerical and analytical

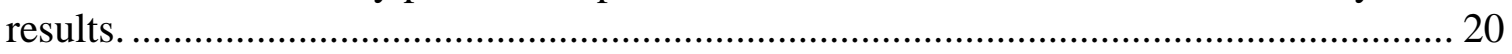

FIGURE 11 - Numerical velocity profiles for cases from TABLE 1 ........................... 21

FIGURE 12 - Zoomed in view of FIGURE 11..................................................... 22

FIGURE 13 - Numerical velocity profiles for cases from Table II compared to the

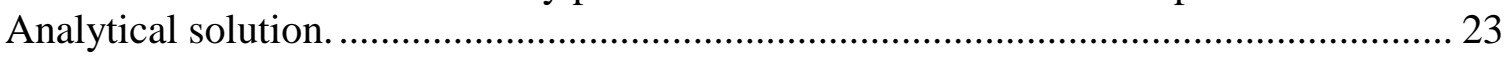

FIGURE 14 - Velocity down the middle of the channel. ............................................ 24

FIGURE 15 - Exponentially stretched grid. ........................................................... 25

FIGURE 16 - Dimensionless exponentially stretched grid numerical velocity profile compared to the analytical velocity profile.......................................................... 25

FIGURE 17 - Fine entrance grid with course exit grid connected by sharing with

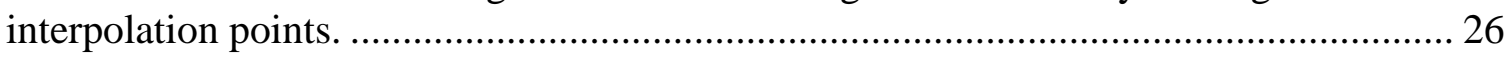

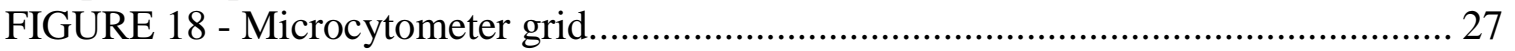

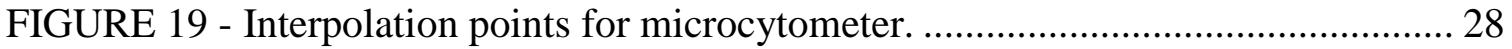

FIGURE 20 - Shared boundaries for the microcytometer. .......................................... 28

FIGURE 21 - Micro-channel boundary conditions. ................................................... 29

FIGURE 22 - Numerical solution for micro-channel in the u direction. ......................... 30

FIGURE 23 - Velocity profile comparison for Analytical, Basic, and Micro-Channel... 31

FIGURE 24 - Microcytometer grid with two cells inserted into the sample stream. ....... 32

FIGURE 25 - Initial position of two cells on the channel when time is equal to zero. .... 32

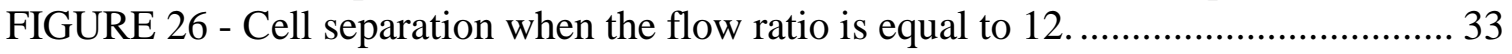

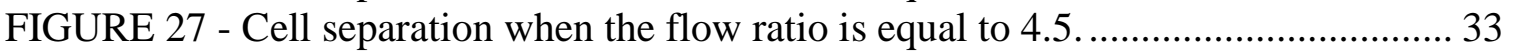

FIGURE 28 - Dye used for measuring the concentration in the sample stream............. 37

FIGURE 29 -Effect of Schmidt number on sample flow concentrations. ....................... 38 


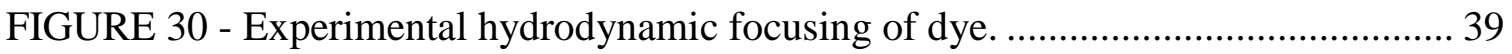

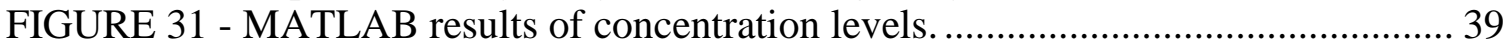

FIGURE 32 - Concentration level, x-position of .5, along the width of the channel. ...... 40

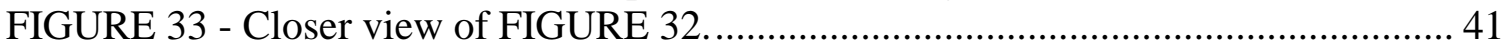

FIGURE 34 - Normalized diffusion along a channel. ................................................ 42

FIGURE 35 - Diffusion down the length of the channel......................................... 43

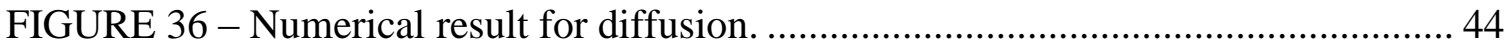

FIGURE 37 - Increased focused stream width as the flow ratio decreases..................... 45

FIGURE 38 - Concentration level comparison between different flow rates $\mathrm{x}=0.5$. ...... 46

FIGURE 39 - Asymmetric flow comparison of different upper and lower sheath stream

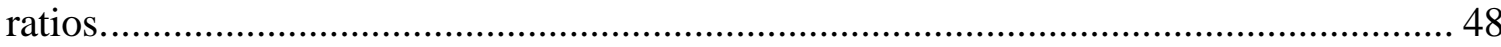

FIGURE 40 - Asymmetric flow position comparison between numerical and analytical.

FIGURE 41 - Three dimensional numerical velocity solution. .................................... 51

FIGURE 42 - Three dimensional numerical velocity solution showing "bullet" profile. 51

FIGURE 43 - Diffusion of the three dimensional channel with a cross section showing

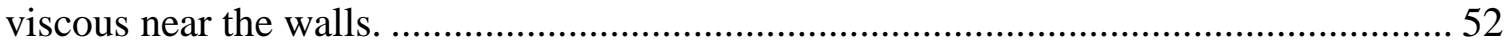

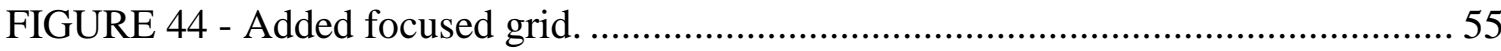




\section{INTRODUCTION}

In this chapter, the relationship of cytometers and microchannels are highlighted. How and why microchannels are able to accurately count and measure cell volume of each passing cell are emphasized. Exploration of hydrodynamic focusing is used to explain why cells will separate. Investigation of different types of hydrodynamic focusing are also studied. Computational fluid dynamics can be used for analyzing microchannels to inform researchers at the University of Louisville about fluid flow, heat transfer, mass transfer, and electrical fields.

Flow cytometers have been around since the 1960s. They are capable of counting and sorting cells as they pass by a detection device. They are used by a variety of disciplines ranging from biological research to drug development. One of the most important diseases that cytometers will detect is blood cancer.

The principle behind the flow within a cytometer consists of a stream of hydrodynamically focused fluid exposed to a single wavelength from a laser beam of light. Hydrodynamically focused fluid is the squeezing of one sample stream and two sheath streams into one smaller stream. Cells, ranging from 0.2 to 150 micrometers in diameter, within the hydrodynamically focused fluid will scatter the beam of light towards detectors placed perpendicular to (side scattering), or in line with (forward 
scattering) the beam of light. As the cells pass the laser beam, fluorescent chemicals within or on the cells are excited and emit longer wavelengths of light than the laser beam. Each detector will show fluctuations in brightness from the fluorescence and scattered light. These fluctuations provide chemical and physical information, such as size or type, about each cell (Hexley \& Elliott, 2010). Forward scattering shows information relating to cell volume, while side scattering shows information based on the nucleus shape and membrane roughness (Abcam, 1998). Hydrodynamic focusing and beam scattering is shown in FIGURE 1 (Abcam, 1998).

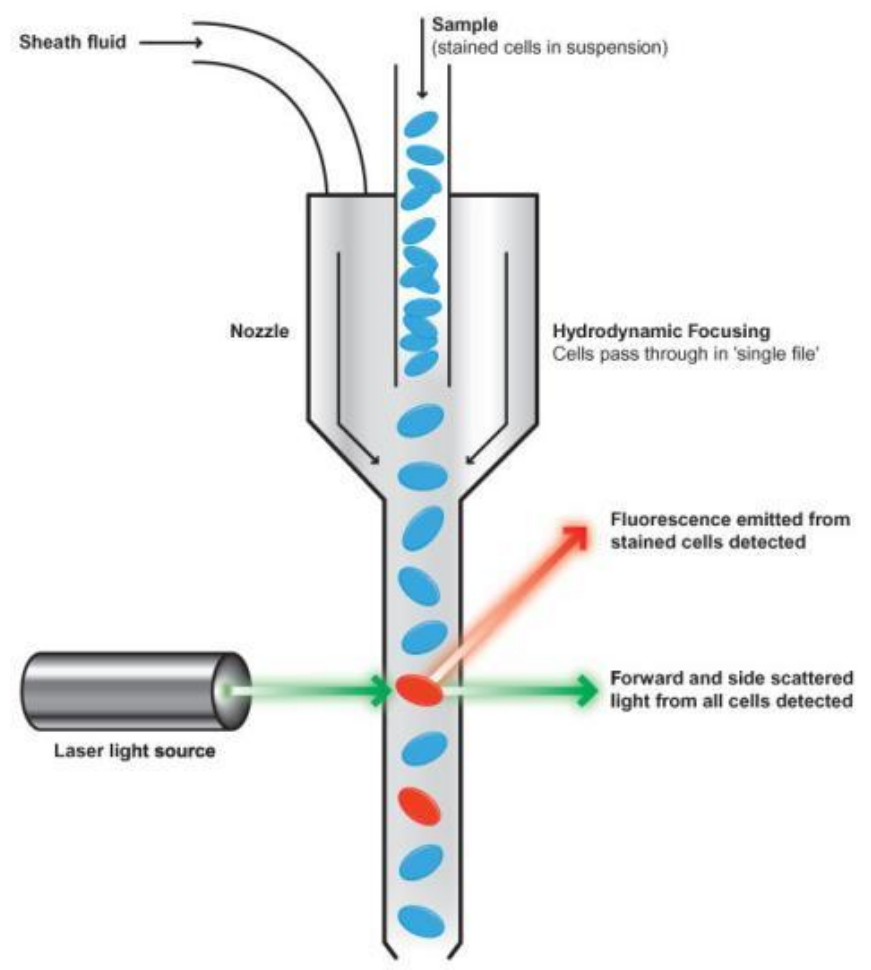

FIGURE 1- Flow process in a cytometer.

Disadvantages of flow cytometers; include (1) the amount of space they occupy in laboratories, (2) they require experts to run, (3) they use vast amounts of power, and (4) are very expensive (Scott, Sethu, \& Harnett, 2008). 
In recent years, attempts to reduce the size of cytometers have led to the development of a device known as a micro cytometer. These smaller versions of cytometers can either retain the original optical detectors for complete cell identification or use the Coulter technique for counting and sorting cells (Scott, Sethu, \& Harnett, 2008). Compared to optical detector based devices, the Coulter technique is more promising because of it increased accuracy, ability to count large amounts of cells, and measure specific cell volume (Houwen, 2003).

\section{A. Coulter Technique}

The Coulter technique is electrically impedance based and has been miniaturized in recent years (Scott, Sethu, \& Harnett, 2008). It is a very valuable concept that determines cell sizes in the biological, medical, and industrial fields (J.H., F., J., P.M., \& M.J., 2004). The Coulter Technique or Principle was introduced in the 1950s. It was developed by the Coulter brothers, Wallace H. and Joseph R. The brothers wanted to create a device that demonstrated repeatability and was more accurate than previous methods of spending 30 minutes looking down a microscope physically counting the cells (Graham, 2003)

Over the following decades the brothers found a way to repeatably count cells. Shown in FIGURE 2 (The Coutler Particle Counter/Sizer) this was accomplished by using two electrodes, one inside a sample tube and the other placed into a larger container. The sample tube had a small needle hole near the bottom and was placed in the larger container along with the second electrode. Ionic fluid was then filled in both containers. Electric current was then passed between the two electrodes and cells 
suspended in ionic fluid were pumped through the needle hole of the sample tube into the larger container (Graham, 2003).

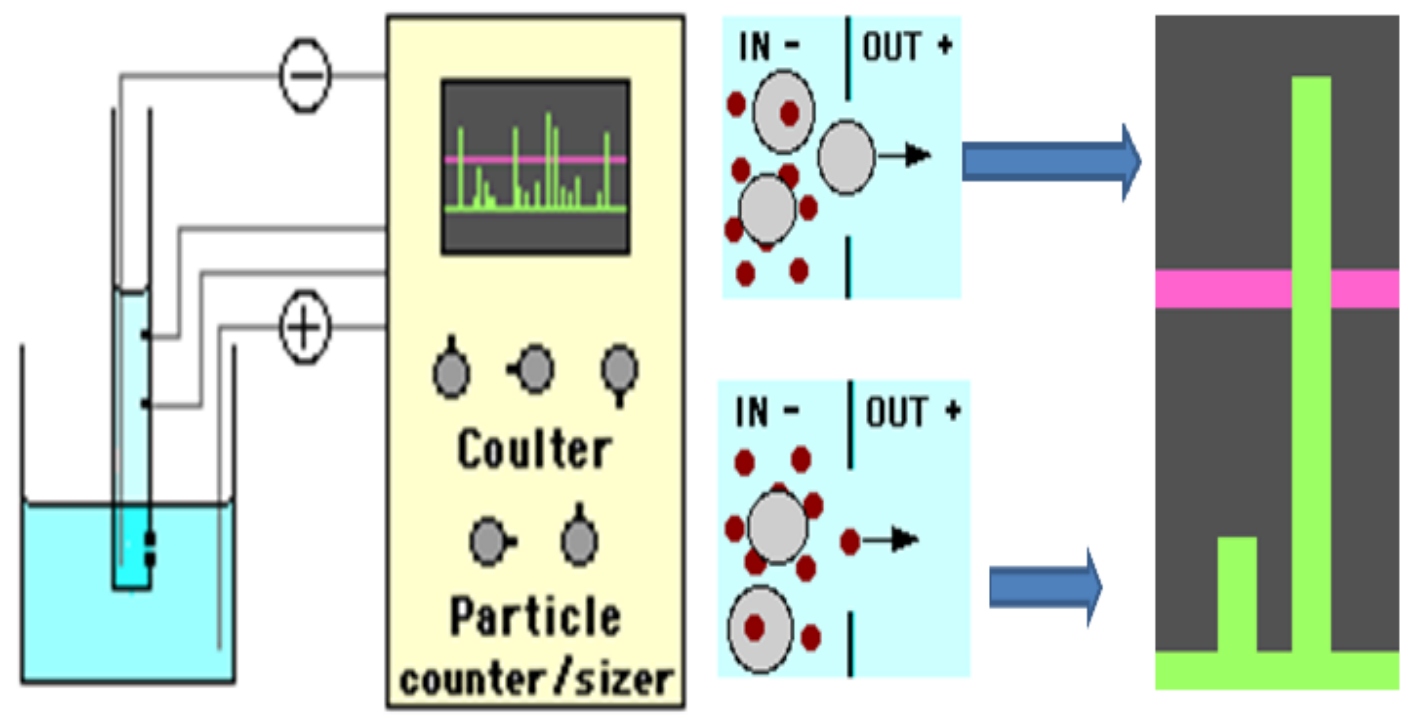

FIGURE 2 - Process of the Coulter Technique.

Even though the Coulter brothers were unaware that cells were insulators at the time, they found that as each cell passed, its volume was proportional to the voltage pulse between the current of the electrodes. With each voltage pulse being recorded the brothers had found a way to accurately and consistently count cells. This technique proved to have an electrical contrast between the ionic fluid and the cells that was 10 times greater than photoelectric methods. This process has proved itself time and again, mainly due to the accuracy, efficiency, convenience, and size of the overall process (Graham, 2003).

In the most recent design of a microchannel at the University of Louisville the theory behind the Coulter technique is used but the process is a little different. Two electrodes and a sample fluid containing cells are used, but the hole for the cells to travel 
through is achieved by the focused stream (Harnett, 2009). The electrodes are placed on top of the focused stream; allowing a constant rate of current into the focused stream. As the cells pass the electric field a change in resistance occurs, producing an impedance signal. The number and magnitude of these spikes can determine the total number and volume of the cells, respectively (Scott, Sethu, \& Harnett, 2008). An experimental signal is shown in FIGURE 3 (Scott, Sethu, \& Harnett, 2008).

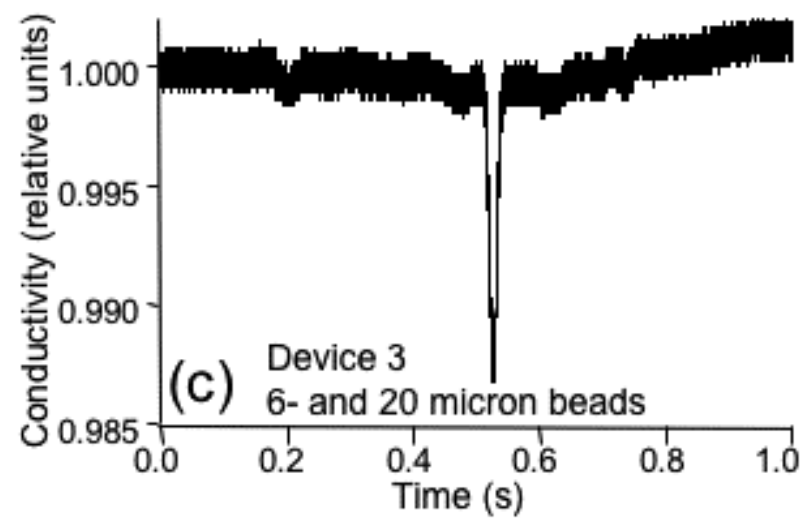

FIGURE 3 - Experimental signal produced when a cell passes the electrodes.

A disadvantage to miniaturizing the Coulter technique is that it can only be used with filtered solutions and the particle size within the fluid needs to be nearly equal to the focused stream width. This is done to increase the change in percentage resistance of the conductive path as each cell passes (Scott, Sethu, \& Harnett, 2008).

\section{B. Hydrodynamic Focusing}

Hydrodynamic focusing employs laminar fluid flow to center and separate cells. As shown in FIGURE 4 separation of the cells is caused by an increase in velocity as three streams, one sample and two sheath streams, fuse into one stream (Edwards, 2009). 


\section{Hydrodynamic Focusing}

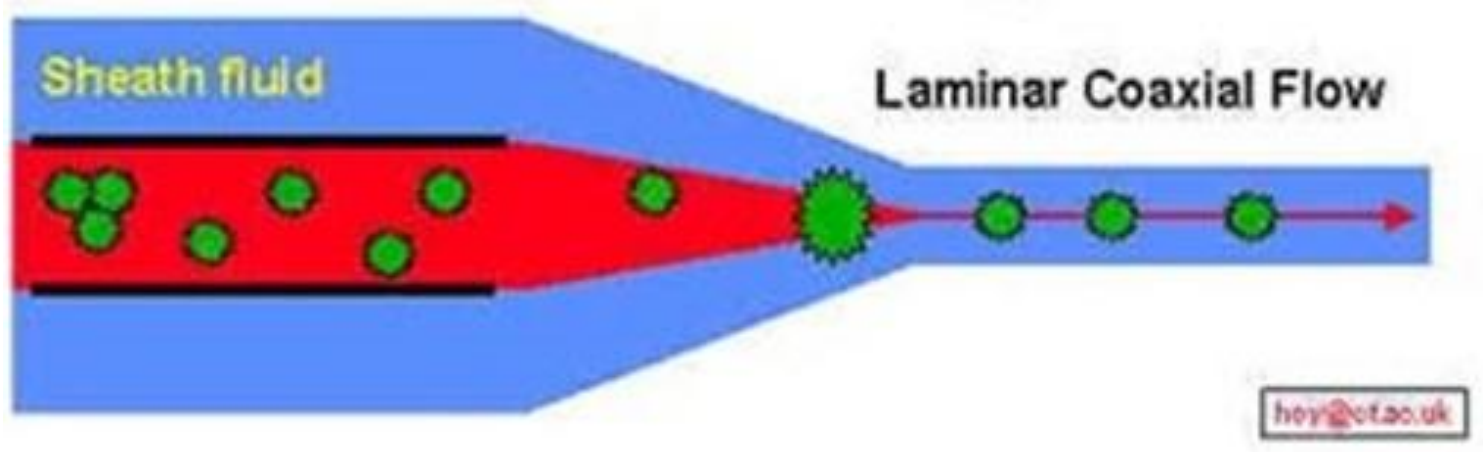

FIGURE 4 - Hydrodynamic focusing (Edwards, 2009).

In the experimental microcytometer the hydrodynamic focusing is achieved by two sheath streams running perpendicular in to the sample stream from the top and bottom directions. This causes the sample stream to squeeze together, known as the focused stream (Harnett, 2009). The sample stream is a conductive fluid and the sheath streams are de-ionized water (Scott, Sethu, \& Harnett, 2008). A schematic of hydrodynamic focusing is shown in FIGURE 5

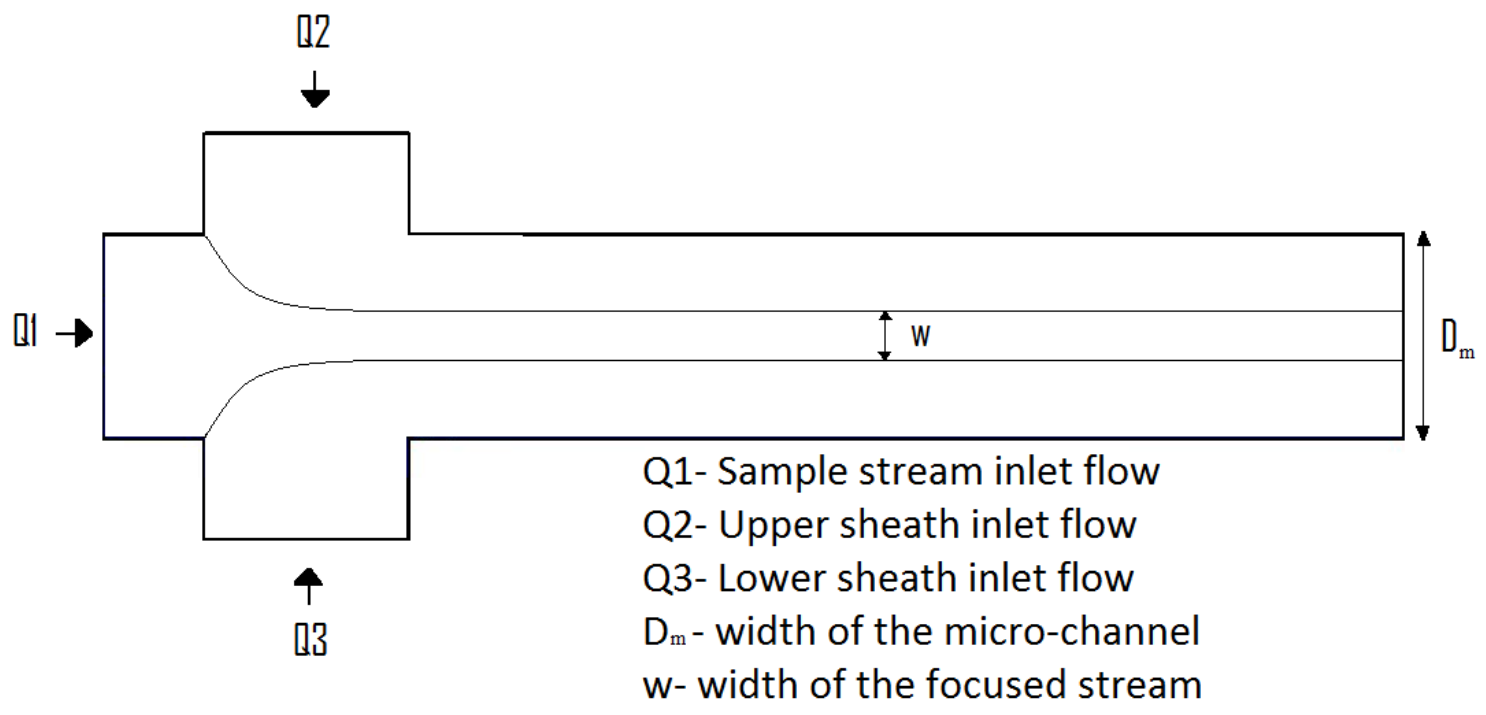

FIGURE 5- Hydrodynamic focusing schematic. 
The use of hydrodynamic focusing in a microcytometer improves the mixing characteristics. This is due to the low Reynolds number and laminar flow of the microcytometer, where the mixing between the two fluids relies on molecular diffusion (Wu \& Nguyen, 2005).

There are two different types of hydrodynamic focusing in microcytometers: asymmetric and symmetric. Asymmetric types use two sheath flows of unequal volumetric flow rates to encase the sample stream. Symmetric types use two sheath flows of equal volumetric flow rates to encase the sample stream (Lee, Chang, Huang, \& Yang, 2005). Symmetric hydrodynamic focusing is shown in FIGURE 6.

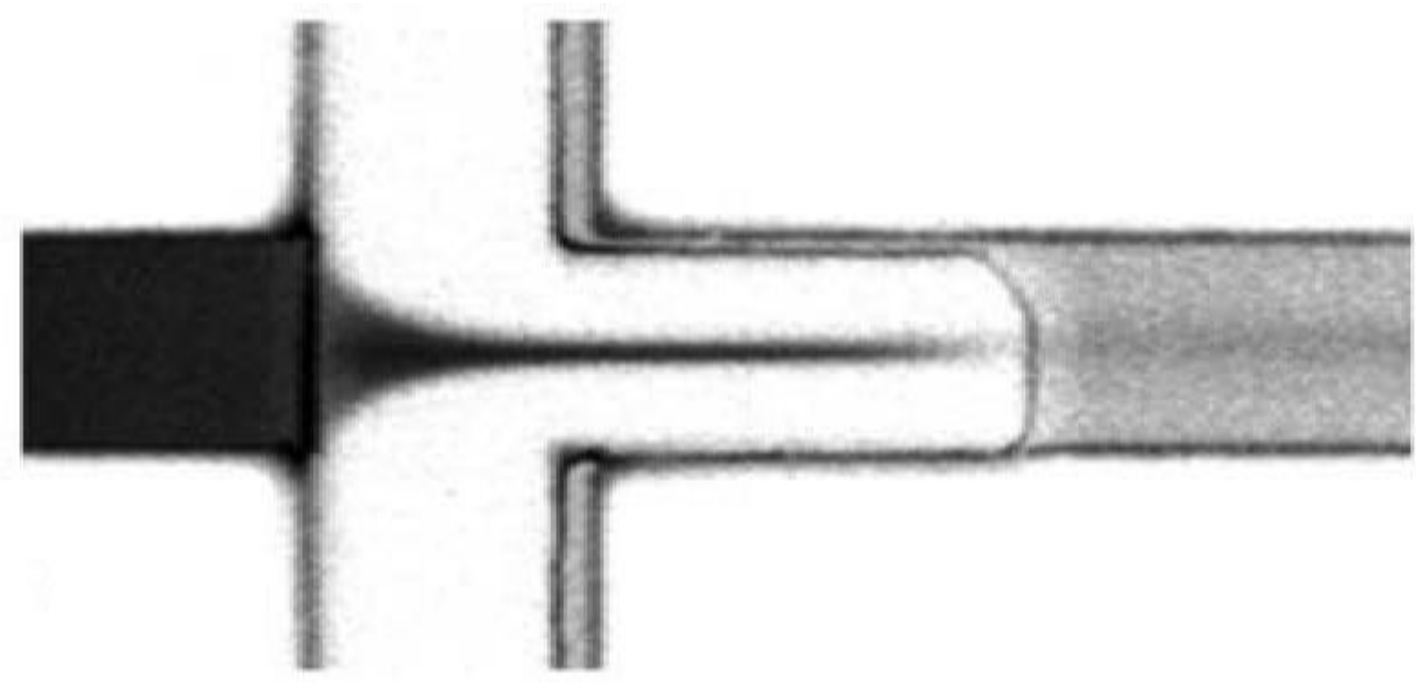

FIGURE 6 - Symmetric hydrodynamic focusing.

Volumetric flow rates for the sheath and sample streams can be controlled by syringe pumps or by creating a pressure drop across the microcytometer (i.e gravity fed). Gravity fed microcytometers are a little more ideal because they allow the flow to be constant, while the syringe based pumps will have pulses in the flow causing a start and stop effect in the flow (Harnett, 2009). 
Different volumetric flow rates of the sheath streams will control the width of the focused stream. Keeping the focused stream roughly the same width as the cell diameter will increase impedance detection as the cells are passed by the electrodes. An increase in detection is caused by the reduced width of the sample stream; this reduction will have an increased change of percentage resistance as the cells pass. For the best impedance signal the focused stream width should be between 6-15 micrometers (Scott, Sethu, \& Harnett, 2008).

To help increase the conductivity and impedance signal, the sample stream uses a dyed aqueous sucrose solution mixed with potassium chloride (KCL). Fluorescent polystyrene micro beads were added to help with visibility and meet the size range (6-20 micrometer) of mammalian/cancer cells. To match the viscosity of the sample stream, the sheath streams used the same aqueous solution minus the presence of KCL. This solution makes the sheath streams less conductive and will have little effect on the impedance signal (Scott, Sethu, \& Harnett, 2008).

Combining the Coulter Technique and hydrodynamic focusing offers researchers the accuracy and speed needed to count cells. Other benefits from this combination include having two different cell diameters in the sample stream with no effects on accuracy of the impedance signal. This combination is also the easiest to employ compared to other methods, however, it is noticeable that lower concentration levels will result in poorer impedance resolution (Shuler, Aris, \& Tsuchiya, 1972).

\section{Microcytometer Device}


Micro-channels have become something of interest over the last couple of decades. Advances in microcytometers and micro fluidic technology have allowed labon-a-chip (LOC) devices and micro total analysis systems (u-TAS) to emerge (Lee, Chang, Huang, \& Yang, 2005). These systems and devices have been used in many fields such as biochemistry, drug delivery, drug screening, biophysics and medial fields, plus biological and chemical assays (Lee, Chang, Huang, \& Yang, 2005). Advantages with microcytometer devices over larger scale models include lower cost, higher throughput, higher performance, and reduced sample and reagent consumption (Lee, Chang, Huang, \& Yang, 2005).

Microcytometers provide a very reliable way of controlling the passage of molecules and samples (i.e. chemical reagents or bio-samples) (Lee, Chang, Huang, \& Yang, 2005). Another important characteristic of these devices is the ability to predict the location of a cell/particle downstream in the channel. This characteristic allows cell/particle samples to be sorted into different groups categorized by size or shape (Walsh, Walsh, \& Davies, 2007).

The experimental microcytometer was created "... by casting silicone elastomer on a two-level mold made from SU-8 negative (MicroChem, Newton, MA, USA), and sealing the elastomer replicas to glass wafers that had been patterned with thin-film metal electrodes." (Scott, Sethu, \& Harnett, 2008).

The mold created channels for the sample and sheath streams with dimensions of 100 X 100 micrometers. Thin-film electrodes used for the Coulter technique are created using $25-50 \mathrm{~nm}$ thick titanium films covered by a $200 \mathrm{~nm}$ thick platinum film. The electrodes were spaced 25 microns apart and placed $3 \mathrm{~mm}$ after the hydrodynamic 
focused area. These types of electrodes are biocompatible and will not be affected by electrochemical corrosion. Area surrounding the channels is a glass substrate that is biocompatible which also allows a clear view of the sample and sheath streams. The glass substrate has a very low conductivity that will help improve the impedance signal (Scott, Sethu, \& Harnett, 2008).

\section{Scope of Research}

University of Louisville researchers are experimenting with microcytometers to count bio-samples containing cancer cells by means of the Coulter technique. The ultimate goal of their research is to accurately count cancer cells with attached nanoparticles. A schematic is shown in FIGURE 7.

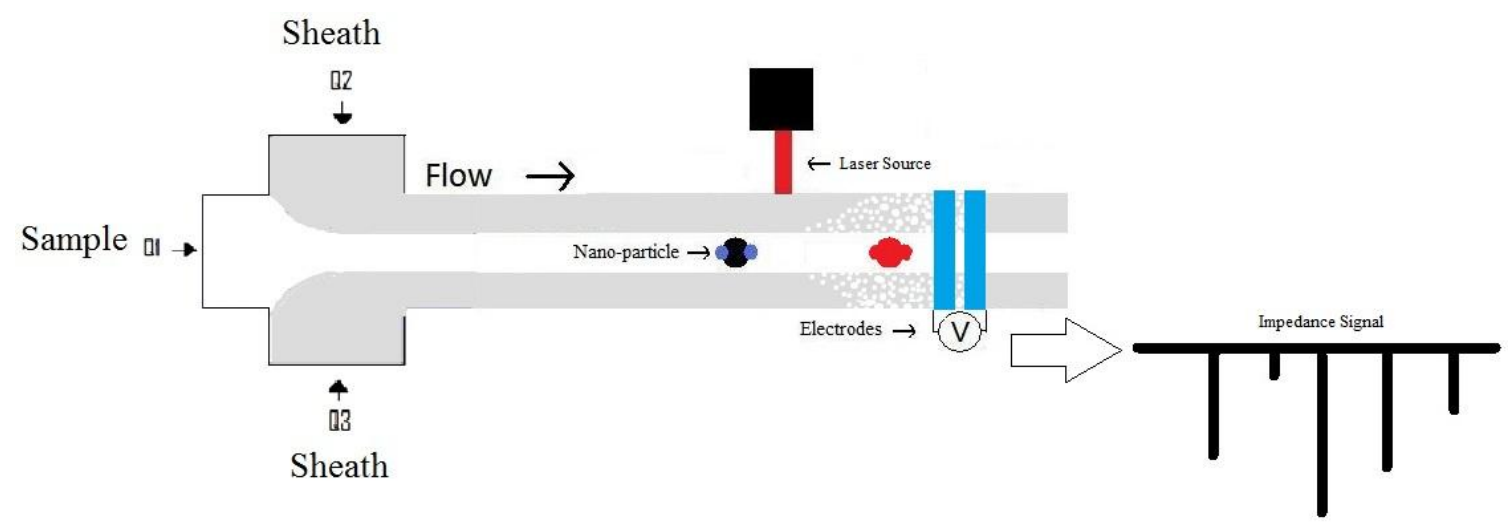

FIGURE 7 - Schematic of multiple domains occurring in microcytometer.

Nano-particles attached to the cancer cells pass through the hydrodynamic focused area of the microcytometer and absorb radiant heat from a laser source. The heating of the cells increases diffusion between the sample and sheath streams. This diffusion will move conductive molecules into the sheath stream allowing the current to flow more 
quickly giving way to a clearer impedance signal and thereby increasing the accuracy of the cell counting (Harnett, 2009). Analysis of the microcytometer will involve fluid dynamics, heat transfer, mass transfer, and electrical properties.

The scope of this research is to characterize fluid flow, mass transfer, and diffusion of the microcytometer by using Computational Fluid Dynamics (CFD) analysis. An in house CFD solver (Overture) will be used to numerically solve a grid that represents the dimensions of the experimental microcytometer. By comparing the velocity profiles of the numerical solution to an analytical result validation of proper results can be shown. Once the analytical and numerical results match, a comparison between flow rates can be tested in order to show which flow rate has the highest concentration level of the sample stream.

For a successful design, it is critical to adjust the flow ratio between the sample and sheath streams to provide high concentration downstream for a better impedance signal at the electrodes. Current practice relies on trial and error, which is not only time consuming but also expensive to furnish manpower and built microcytometers.

CFD will be used to identify a flow ratio that provides the highest concentration near the electrodes. Furthermore, CFD techniques will help understand the mechanisms involved in the microcytometer, particularly the effects of different flow ratios on the sample stream and fluid/solid motion problem. CFD techniques can also investigate the diffusivity effect on the concentration of the sample stream and three dimensional effects. 


\section{FLUID FLOW IN MICROCYTOMETERS USING NUMERICAL SIMULATION}

In this chapter, an explanation of governing equations used to solve fluid flow and mass transfer, boundary conditions for each grid and what/how Overture creates and interpolates grids.

The flow behavior can be simulated by solving the Navier-Stokes equations together with the mass transfer equations. For incompressible flow, the governing equations are:

$$
\begin{gathered}
\rho\left(u_{t}+u \cdot \nabla u-f\right)=-\nabla p+\nabla \cdot \sigma \\
\nabla \cdot u=0 \\
m_{i, t}+(u \cdot \nabla) m_{i}=\nabla \cdot\left(D \nabla m_{i}\right)
\end{gathered}
$$

where $\rho$ is the density, $u$ is the velocity, $f$ denotes the body forces (gravitational force) per unit mass, $p$ is the pressure, $\sigma$ is the shear stress and equals $\mu\left(\nabla \cdot u+\nabla \cdot u^{T}\right), \mu$ is the kinematic viscosity, $m_{i}$ is the mass fraction of the ion in the fluid, $D$ is the mass diffusivity. The incompressible Navier-Stokes equations are solved with the pressurePoisson method. The equations are discretized in space with fourth-order accuracy using central difference method. A method of lines approach is used to solve the equations in time. After discretizing the equations in time, the system of ordinary differential 
equations are solved using explicit time-stepping procedures such as the Runge-Kutta method (Henshaw, 2004).

Boundary conditions applied to the channel are

$\underline{\text { noSlipWall }}$

$$
\begin{array}{ll}
\mathrm{u}=\mathrm{g} & \text { velocity specified } \\
\nabla \cdot u=0 & \text { divergence zero }
\end{array}
$$

\section{InflowWithVelocityGiven}

$$
\begin{array}{ll}
\mathrm{u}=\mathrm{g} & \text { Velocity specified } \\
\partial_{n} p=0 & \text { Normal derivative of the pressure zero }
\end{array}
$$

outflow

extrapolate $u$

$$
\alpha p+\beta \partial_{n} p=g \quad \text { Mixed derivative of } \mathrm{p} \text { given }
$$

Overture uses an object oriented framework that solves partial differential equations (PDE's) (Henshaw, 1998). Since Overture is an object oriented framework it is capable of solving complex domains, multi-physics parameters, and moving grid problems; this is done by creating a structured or a collection of structured grids. Structured grids used by Overture gives an advantage over other solvers because the object oriented framework reduces the learning curve, code duplication, and interoperability of application software. Other advantages when using Overture are easier use of complex geometries for moving grids in serial or parallel computations, and better understanding of numerical simulations for research in industrial and scientific applications (Henshaw, 1998). 
For Overture to numerically solve PDEs of fluid mechanics, approximations must be made to the PDEs. These approximations are used to rewrite PDEs as algebraic equations by transforming PDEs to finite difference equations (FDEs). These FDEs are solved at discrete points within a grid. Note that when the equations are converted and transformed from the original PDEs, the form of the original equation will be the same as the transformed equation; in such that if the original is parabolic, then the transformed equation will also be parabolic (Hoffmann, 1989).

In the Coulter counter, a tagged cell moves inside a channel that is subjected to external forces and torques, leading to a moving boundary problem. Moving boundary problems require special techniques to automatically adjust the computational grid because the computational domain is not fixed, and may not be known a priori. This is solved by employing the overlapping moving grid technique. An overlapping grid consists of a set of logically rectangular grids that cover a region and overlap where they meet. Interpolation conditions are used to connect the solutions to different component grids. Typically, boundary fitted curvilinear grids are used near the boundaries while Cartesian grids are used to handle the bulk of the domain as the background (Henshaw, 2008). FIGURE 8 shows an overlapping grid (Henshaw, 2004). 

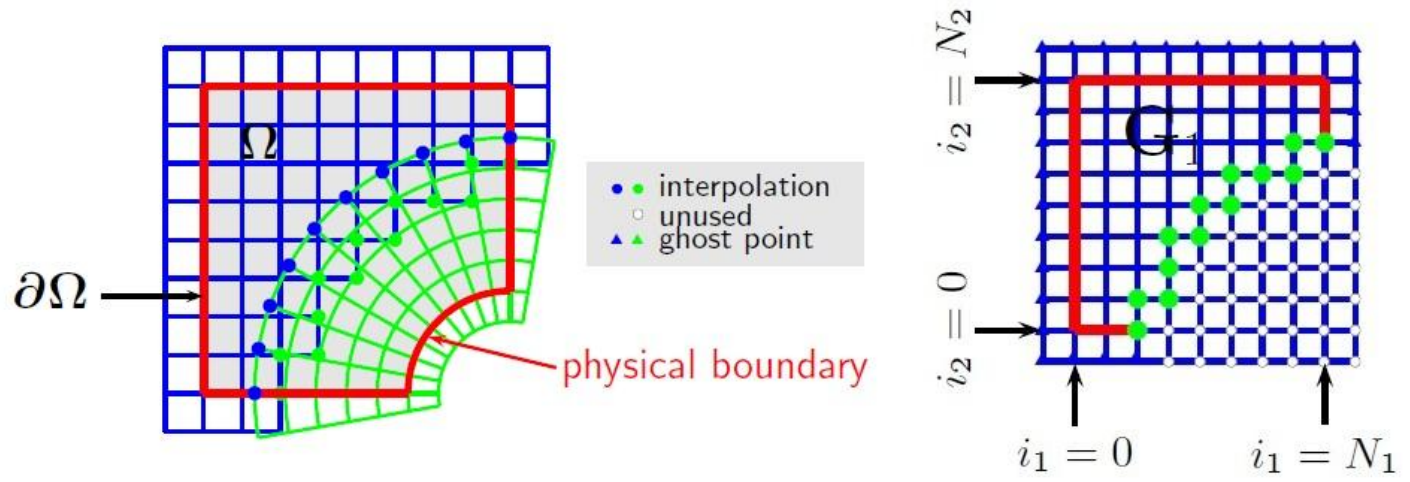

FIGURE 8 - A simple overlapping grid. Left: the overlapping grid, right: overlapping grid in the parameter space.

The Cartesian grid in blue covers the rectangular computational domain; the boundary fitted grid in green surrounds the circular cell.

In this example, the cell grid cuts a hole in the Cartesian grid, resulting into a number of unused points. The governing equations are discretized on the discretization points and interpolation points. Solution values on the interpolation points are obtained using a tensor-product Lagrange interpolant. As discussed in (Henshaw, 2008), the overlapping grid technique has many desirable characteristics in modeling problems with complex geometries. First complex domains can be represented by smooth grids, this is important when obtaining accurate approximations to PDEs and boundary conditions. Second, because overlapping grids mainly consist of Cartesian grid cells, it retains speed and requires low memory usage. Third, it can handle moving boundary problems in complex three-dimensional regions. This is particularly important when considering three dimensional flows.

\section{A. Ogen and Grid Generation}


Ogen, subsidiary application of Overture can be used to generate computational grids for solving simulations. For grid generation the following rules are generally followed; (1) grids lines cannot cross each other unless interpolation points are used, (2) a grid should have very little skewness, i.e., grid lines should be perpendicular or near perpendicular, and (3) stretching is needed in areas of high flow gradients (Hoffmann, 1989).

Three techniques are widely used for grid generation; (1) partial differential equation methods, (2) conformal mappings based on complex variables, and (3) algebraic methods. These techniques are either adaptive or fixed; where a fixed grid is independent of the actual solution of the governing equations; this grid will also remain fixed and is created prior to solving the governing equations. An adaptive grid typically changes with the solution (Hoffmann, 1989).

A sample grid for channel flow simulation was created in Ogen. To create the basic grid, Ogen uses a GUI setup that is Linux based where line commands are needed to create an object or grid. The command file used for creating the basic grid is shown in Appendix I, the basic grid is shown in FIGURE 9.

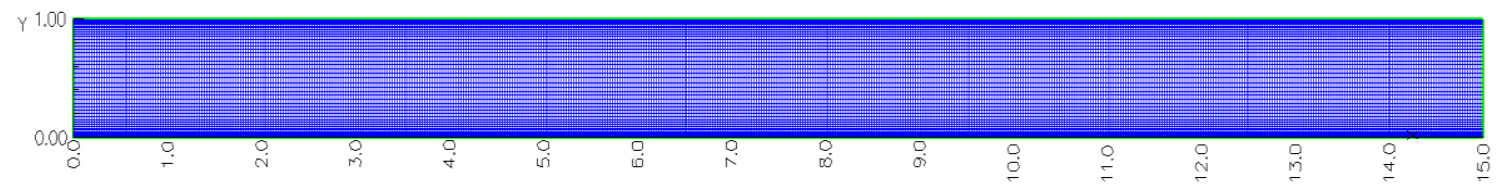

FIGURE 9 - Basic channel grid created in Ogen.

In the grid shown in FIGURE 9, stretching is applied on y-direction grid lines toward the walls of the channel. 
Stretching grid lines causes unequal spacing throughout the channel where the boundary conditions create complications in the Finite Difference Equations (FDEs); this is due to approximations of the non-equal step sizing between grid lines. Also, the boundary conditions have such an impact on the solutions of the equations; inaccuracies due to the interpolations are present in the greatest sensitivity areas (Hoffmann, 1989). 


\section{CHANNEL FLOW SIMULATION}

In this chapter, flow behavior inside a single channel is studied. Comparing the velocity profile of the single channel's numerical solution to the velocity profile of the analytical solution will validate the fluid solver. Next, fluid flow in a channel with three inlets is investigated. Finally the impact of different flow ratios on a moving cell within the microcytometer is explored.

\section{A. Flow Through A Single Channel}

A Reynolds number of 100 was used for the analysis of a single channel, where the Reynolds number is based on the mean inlet velocity and channel height. The dimensionless height of the channel is 1 and the mean inlet velocity is 1 , leading to a dimensionless kinematic viscosity of .01 . The kinematic viscosity is represented by $m u$ in the code for solving the channel grid shown in Appendix II.

Fluid flow in a channel, the velocity profile is governed by the dimensional analysis of the isothermal Navier-Stokes equations for mass and momentum conservation; which is best described by a steady and incompressible state. This analysis has shown that the only governing parameter is the Reynolds number (Okiishi, 2006). The Reynolds number is defined by EQUATION 4. 


$$
\operatorname{Re}=\frac{\rho V D_{h}}{\mu}
$$

Where, $\mu$ is the kinematic viscosity, $\mathrm{V}$ is the inlet velocity, $\mathrm{D}_{\mathrm{h}}$ is the hydraulic diameter, and $\rho$ is the density of the fluid (Okiishi, 2006).

The Reynolds number can be used as a relational factor between the experimental device and the numerical simulation. This is achieved because dimensional analysis shows that when the Reynolds numbers are the same, the fluid will behave in the same manner. In other words, the diameter or length of the channel does not have to match (Okiishi, 2006).

Flow becomes fully developed at some distance downstream of the inlet. This is known as the entrance length and is found using EQUATION 5.

$$
l_{e} / D_{h} \approx .06 \times \operatorname{Re}
$$

Where $l_{\mathrm{e}}$ is the entrance length, $\mathrm{D}_{\mathrm{h}}$ is the hydraulic diameter, and $\mathrm{Re}_{\mathrm{h}}$ is the Reynolds number. This equation is a well known correlation reported by White (1991) and Schlichting (1979) of laminar viscous flow (Okiishi, 2006). According to EQUATION 5 the dimensionless entrance lengths for a microcytometer should be between .6 and 6 . This is based on microcytometer running at Reynolds numbers between 1 and 10 (Scott, Sethu, \& Harnett, 2008).

An analytical solution for the velocity distribution for a Newtonian fluid between two parallel plates is found using EQUATION 6. 


$$
u=\frac{3 V}{2}\left[1-\left(\frac{y}{h}\right)^{2}\right]
$$

Where $\mathrm{V}$ is the inlet velocity, $\mathrm{y}$ is a point along the vertical axis, and $\mathrm{h}$ is half the width of the channel (Okiishi, 2006).

To view and analyze the numerical solution a sub program within Overture called aero was used. The command file for producing a numerical velocity profile in aero is shown Appendix III. A comparison between numerical and analytical velocity profiles are represented in FIGURE 10.

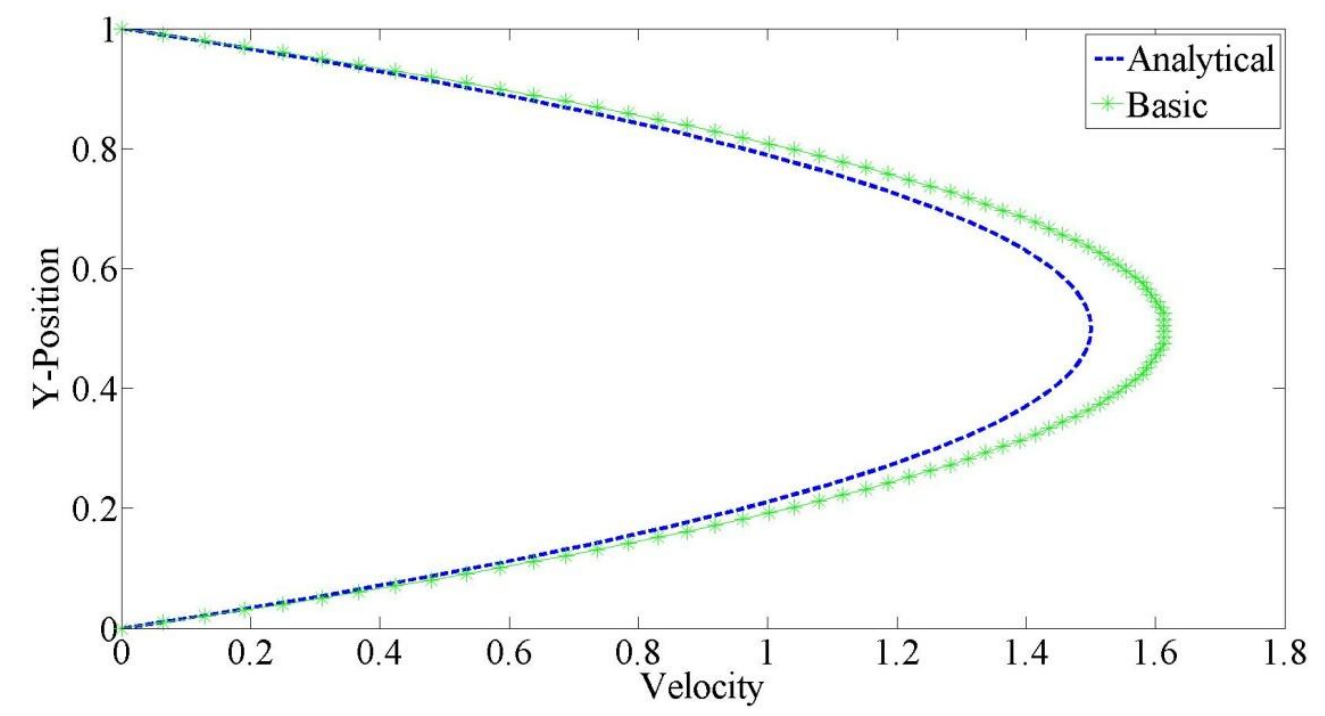

FIGURE 10 - Velocity profile comparison between the basic numerical and analytical results.

Analysis of FIGURE 10 shows a numerical velocity profile that does not match the analytical velocity profile. This is due to the insufficient number of grid lines in the channel grid.

Before continuing to studying the flow physics of the channel, grid sensitivity analysis is performed to understand the impact of the channel grid on the numerical 
solution. Grid sensitivity can be used to reduce the overall error between the numerical velocity profile and analytical velocity profile. The process of grid sensitivity is to improve the percentage error between each solution by stretching or adding grid lines in either direction.

To understand how the numerical solution changes with different grid lines, a first attempt will start by changing the $x$-direction grid lines, these cases are shown in TABLE

I.

\section{TABLE I}

NUMERICAL CASES FOR INCREASING X-DIRECTION GRID LINES

\begin{tabular}{|c|c|c|c|c|c|}
\hline Case & 1 & 2 & 3 & 4 & 5 \\
\hline Y-Direction & 32 & 32 & 32 & 32 & 32 \\
\hline X-Direction & 850 & 950 & 1100 & 1300 & 1450 \\
\hline
\end{tabular}

FIGURE 11 shows numerical velocity profiles for each case in TABLE I.

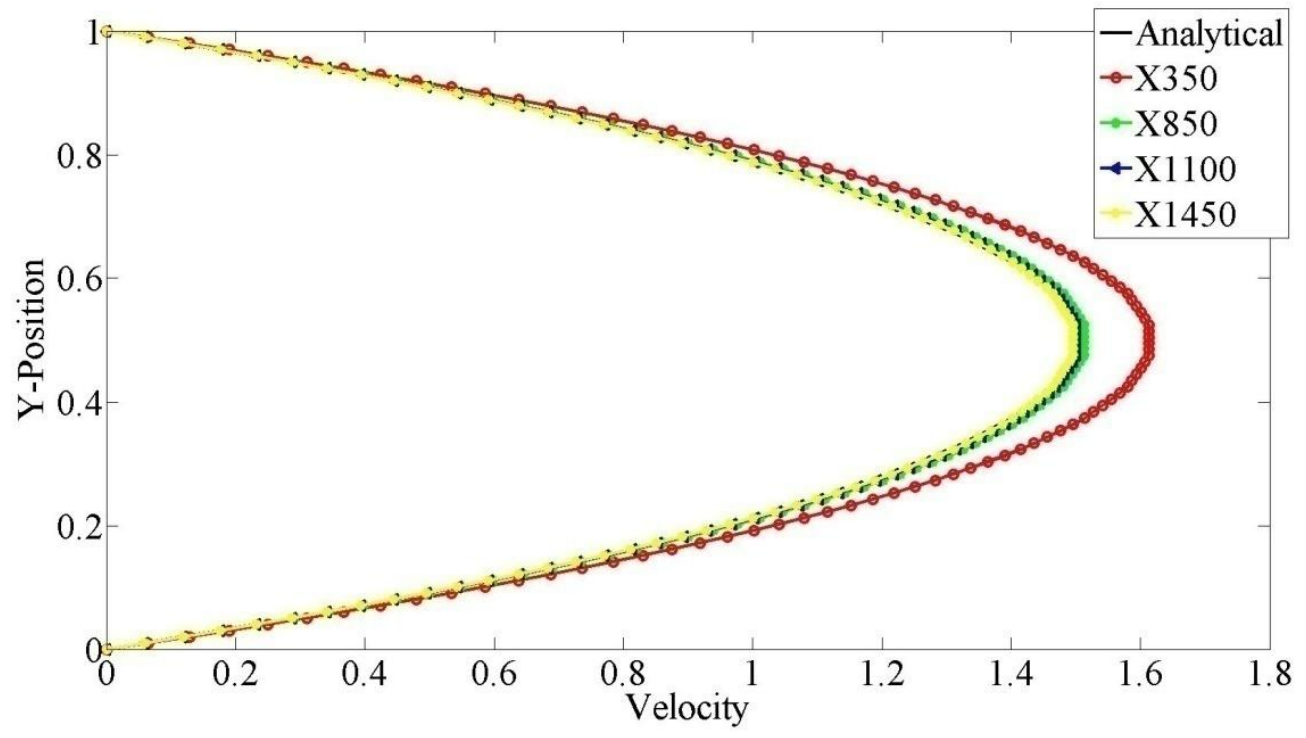

FIGURE 11 - Numerical velocity profiles for cases from TABLE 1

A closer view of FIGURE 11 is shown in FIGURE 12. 


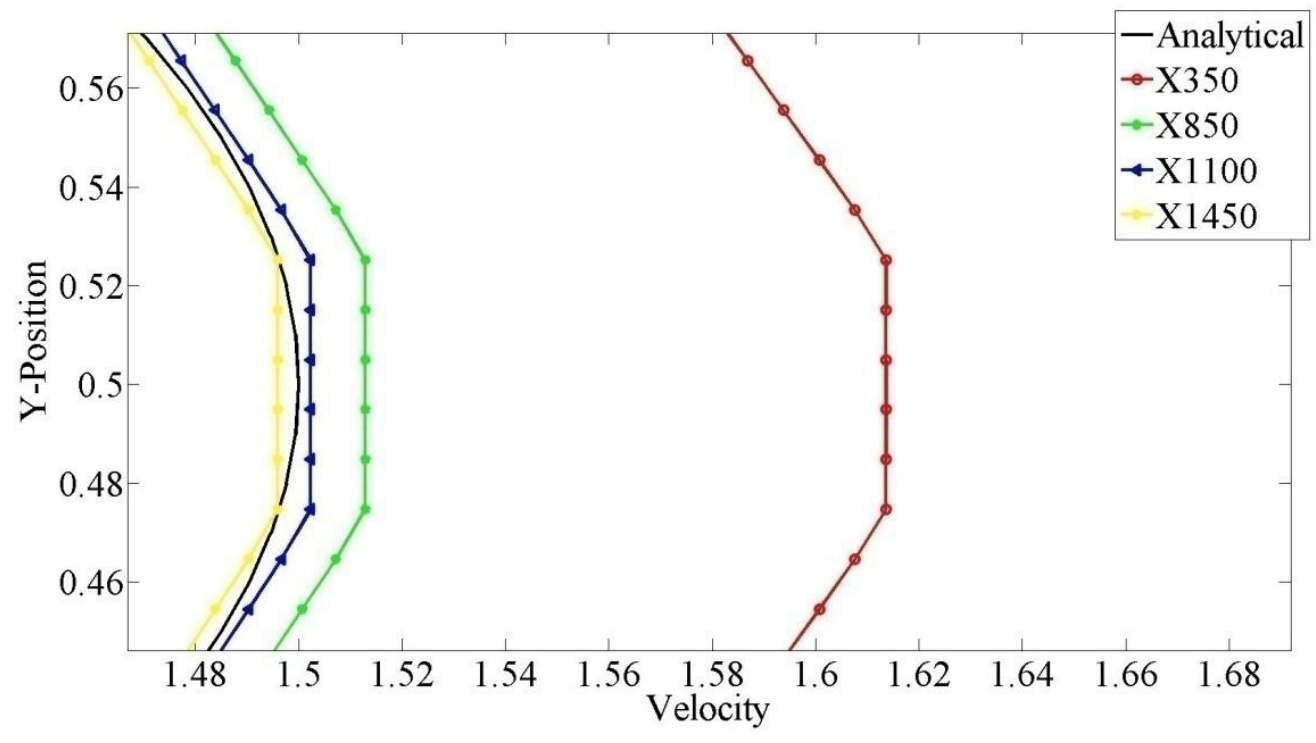

FIGURE 12 - Zoomed in view of FIGURE 11.

Evaluation of FIGURES 14 and 15 determines that as the x-direction grid lines are increased the numerical velocity profile converges onto the analytical solution. Matching the analytical to the numerical solution was ultimately achieved. The time to solve case number 3 from TABLE 1 took a week to solve. This is not acceptable for the size and the number of grid points of the microcytometer grid.

Our second attempt is to increase the number of grid points in both the $\mathrm{x}$ and $\mathrm{y}$ direction. TABLE II shows this progression.

TABLE II

CASES INCREASING GRID LINES BY 30\% IN HORIZONTAL AND VERTICAL DIRECTIONS

\begin{tabular}{|c|c|c|c|c|c|}
\hline Case & 1 & 2 & 3 & 4 & 5 \\
\hline Y-Direction & 210 & 280 & 365 & 585 & 940 \\
\hline X-Direction & 60 & 70 & 80 & 100 & 120 \\
\hline
\end{tabular}


FIGURE 13 shows numerical velocity profiles using the grids from TABLE II compared to the Analytical solution.

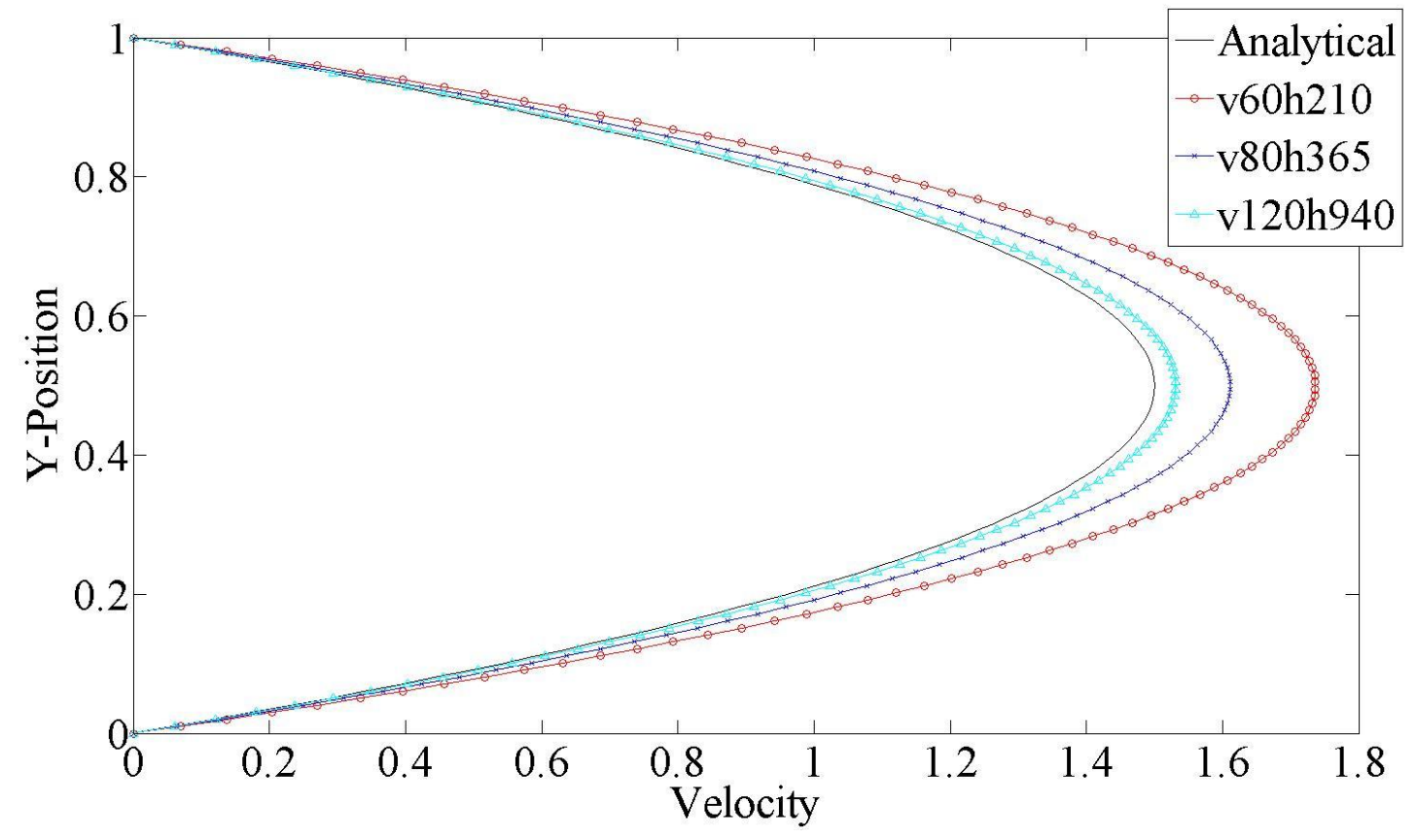

FIGURE 13 - Numerical velocity profiles for cases from Table II compared to the Analytical solution.

Observation of FIGURE 13 shows the numerical solutions slowly converging on the analytical solution. Since the numerical velocity profile is greater than the analytical, mass is not being conserved. Continuing on with this approach would eventually give a numerical velocity profile that matched the analytical solution and conserved mass. The processing time to complete these cases would be on the order of three or four weeks. Obviously this is far longer than the first attempt and will not be suitable. A closer view of FIGURE 13 also shows that the final grid should have grid lines in the y direction greater than 60 because this will give a smooth numerical velocity profile throughout the entire channel. 
Third attempt to match the Analytical solution resulted in going back to the drawing board to understand what is happening within the channel. The streamwise velocity profile at the centerline of the channel is shown in FIGURE 14,

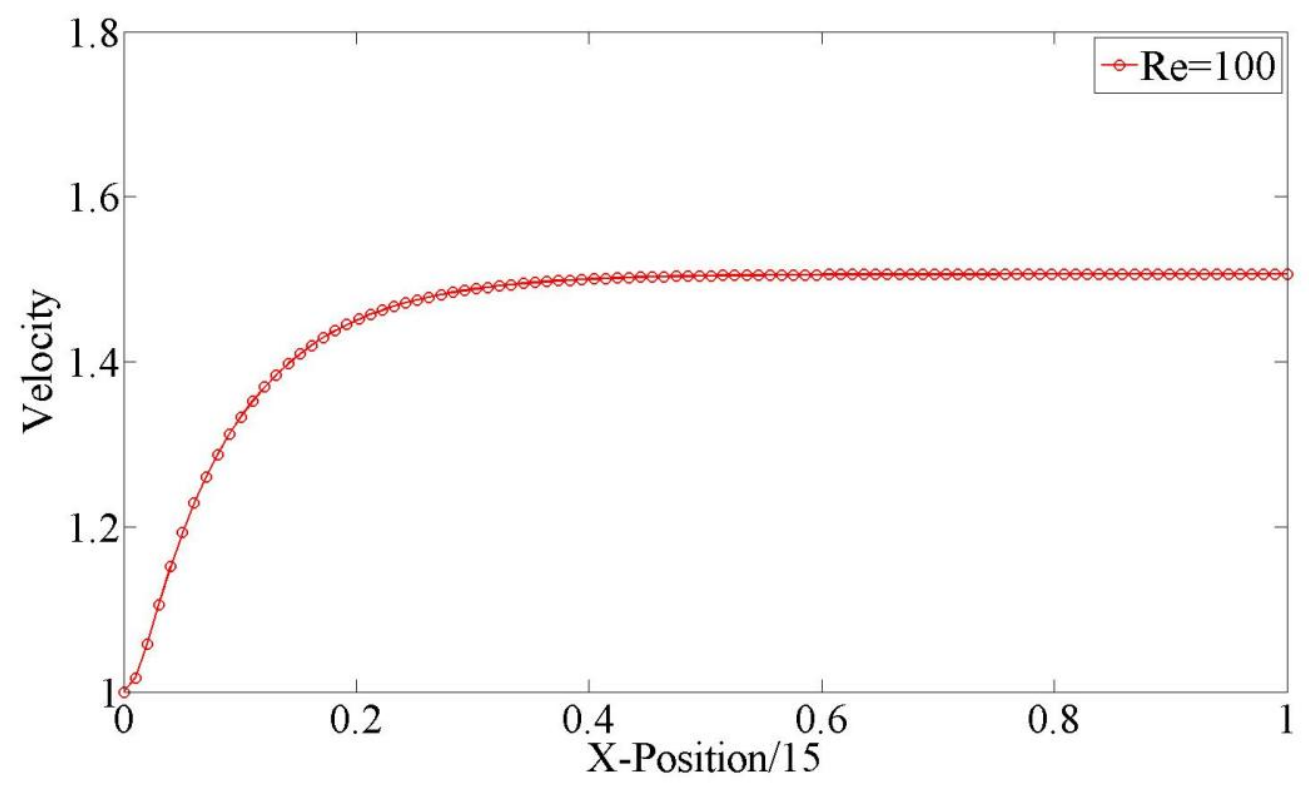

FIGURE 14 - Velocity down the middle of the channel.

Notice that the velocity experiences a quick change near the entrance, but is almost constant downstream. This observation motivates the use of a fine grid near the inlet and a coarse grid downstream. The previous two attempts used uniform grid spacing in the $\mathrm{x}$ direction. With uniform spacing, a larger number of grid points were needed to capture the velocity variation near the entrance.

Exponential grid stretching is applied in the x-direction, shown in FIGURE 15. Creating the exponential grid is shown in the command lines of Appendix IV. 


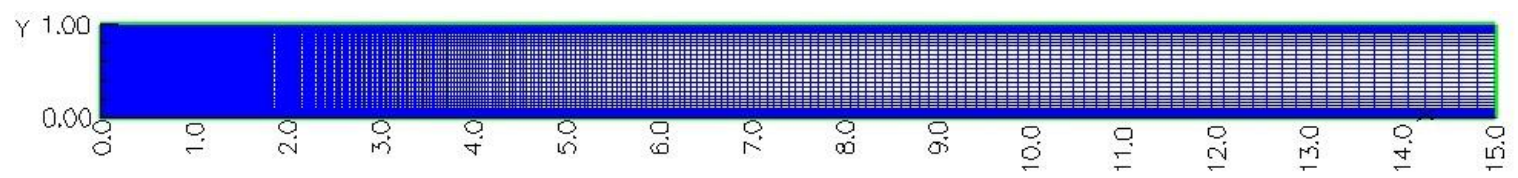

FIGURE 15 - Exponentially stretched grid.

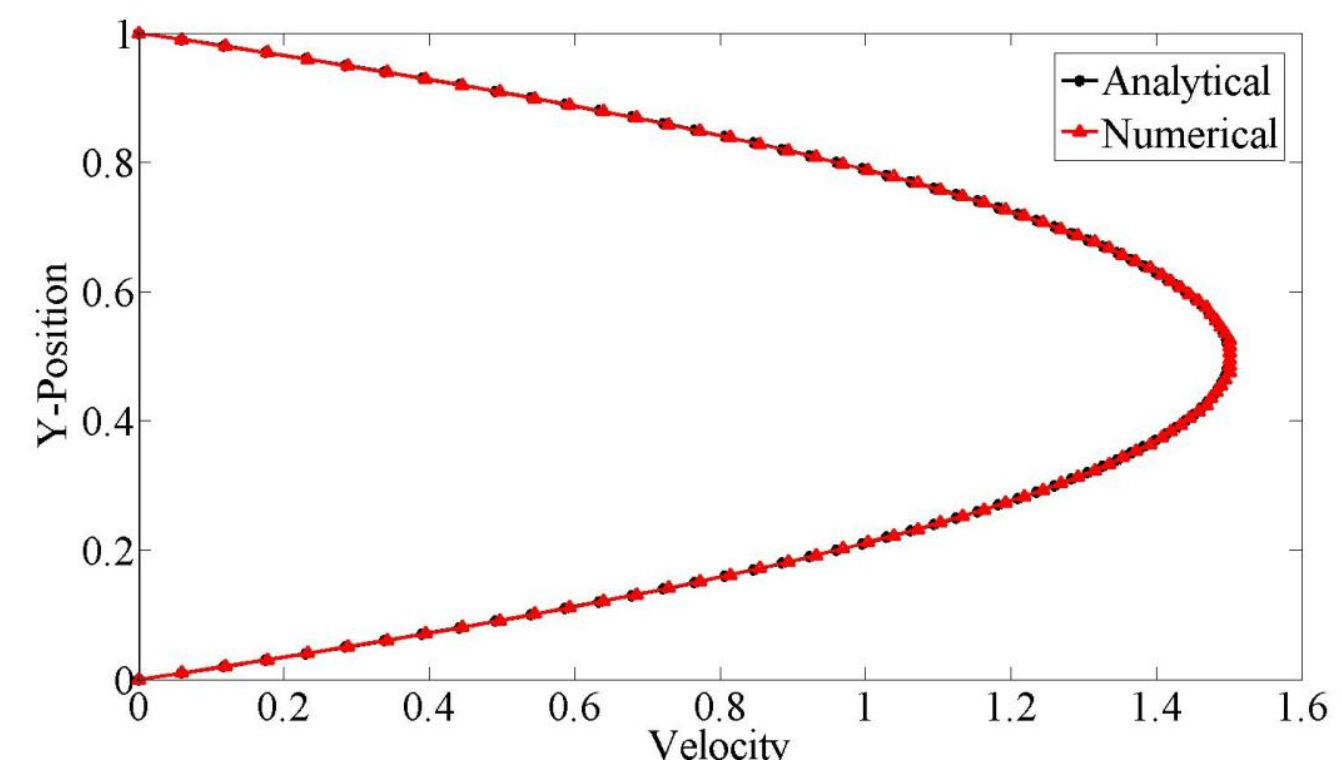

FIGURE 16 - Dimensionless exponentially stretched grid numerical velocity profile compared to the analytical velocity profile.

Shown in FIGURE 16, the exponential stretching of the $\mathrm{x}$-direction grid lines predicts the velocity profile in an effective and accurate manner. However, stretching potentially has a problem when trying to study the cell motion within the channel. With exponential stretching the grid spacing towards the outlet of the channel can be much larger than the cell diameter, resulting in an overlapping grid failure. To solve the problem two uniform grids with different grid densities can be used; one fine grid for the front of the channel to capture the large velocity gradient and one coarse grid for the rest of the channel. A coarse grid is used because the velocity after the entrance length is constant. Due diligence is needed to ensure the coarse can be used to simulate the cell 
motion. The command line to create this channel is shown in Appendix V. The fine and coarse grid channel created in Ogen is shown in FIGURE 17. There were a couple of changes to each grid in order for them to work together. The right side of the first grid and the left side of the second grid needed to be set as interpolation sides, these points are seen in FIGURE 17.

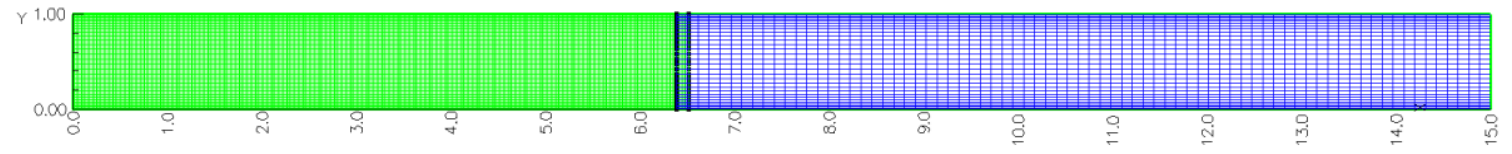

FIGURE 17 - Fine entrance grid with course exit grid connected by sharing with interpolation points.

Interpolation points allow the numerical solution being solved to be passed from one grid to the other. Further information on interpolation points can be found with (Henshaw, 2004).

Comparing the exponentially stretched grid and the shared grid shows that they both match the analytical solution, however the exponentially stretched grid does require 150 less $\mathrm{x}$ directional grid lines; giving the exponentially stretched grid a faster processing time. However, the small amount of increased processing time for the shared grid is better than trying to deal with a failed grid when cells are present.

\section{B. Microcytometer Grid: A Three Inlet Channel}

As mentioned in Chapter1, the microcytometer design consists of three inlets, two for sheath steams and one for a sample stream. The microcytometer grid was created with the command code shown in Appendix VI. Compared to the single inlet channel 
case, two additional blocks above and below the main channel are added, where the main channel now represents the sample stream.

These two grids have the same width as the sample stream, where the top line of the lower sheath and bottom of the upper sheath are set as interpolation points to allow flow into the sample stream. Also a sharing option was turned on. Sharing between the grids helps tell Overture the sides of the upper and lower sheath grids share a side of the sample stream, this option informs the software Overture that there should be a solid connection between each grid. The microcytometer grid, microcytometer grid with interpolation points, and microcytometer grid with shared values are displayed in FIGURE 22-24.

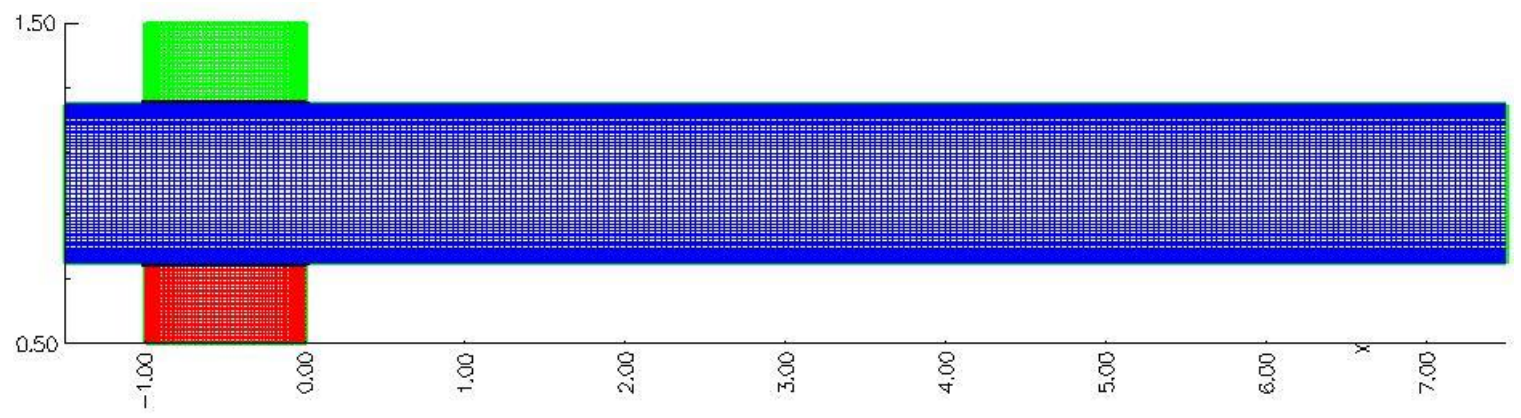

FIGURE 18 - Microcytometer grid. 


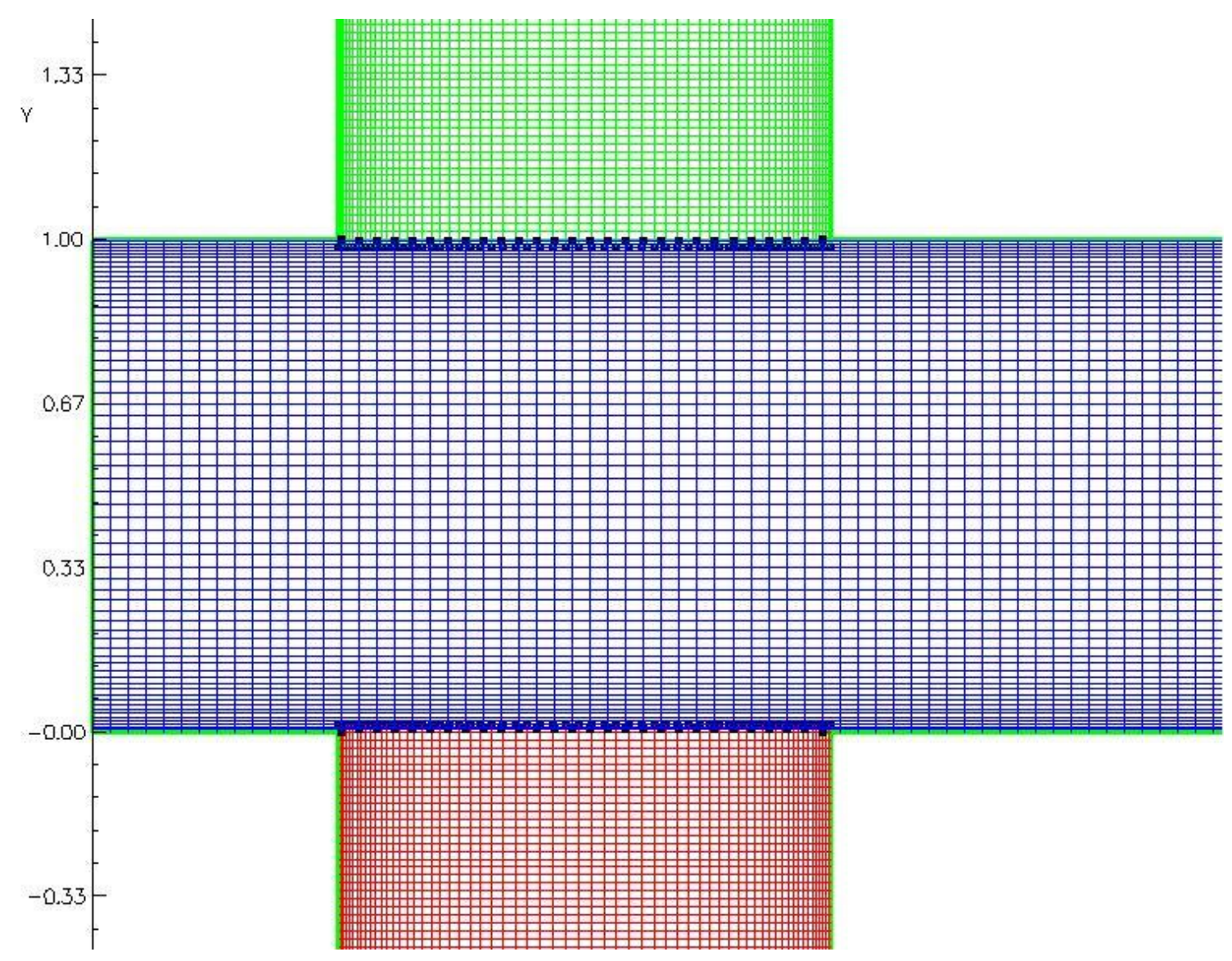

FIGURE 19 - Interpolation points for microcytometer.

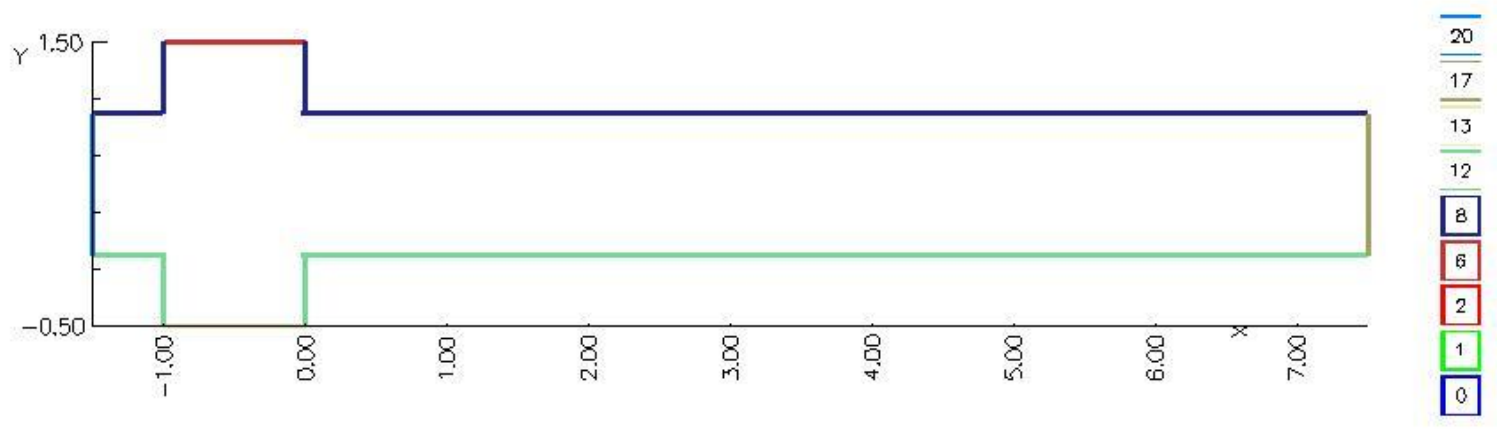

FIGURE 20 - Shared boundaries for the microcytometer. 
To numerically solve the microcytometer a subsidiary application called Cgins was used. The dimensionless inlet mass flow rates of the sample, upper sheath, and lower sheath streams need to add up to one; this will ensure that when the three streams merge into the main channel the overall mass flow rate is the same as the single channel case. The mass flow rate of the sample steam is 0.04 while the mass flow rate of the other two sheath streams is 0.48 . Last change is the switch from a Reynolds number of 100 to a Reynolds number of 1 , this change will represent the experimental microcytometer better.

Cgins code for the microcytometer is along the same basis as the basic codes except for the addition of the inflow boundary conditions for the sheath streams. The microcytometer Cgins code can be seen in Appendix VII. Other notes on the microchannel code are the direction of the sheath flow boundary conditions. The inlet flow for the upper sheath stream is in the $\mathrm{v}$-direction moving down, giving $\mathrm{v}$ a negative value. The lower sheath stream is in the opposite $\mathrm{v}$ direction; giving $\mathrm{v}$ a positive value. The boundary conditions are shown in FIGURE 21, where red represents an inlet, blue represents an outlet, and green is a no slip wall.

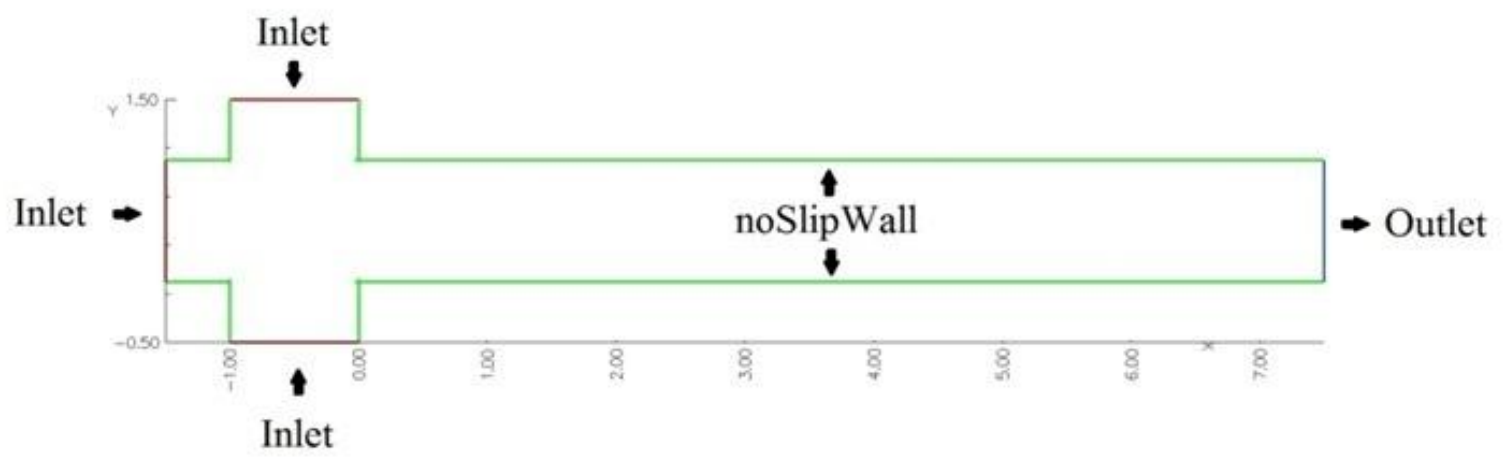

FIGURE 21 - Micro-channel boundary conditions. 
Grid sensitivity analysis was performed. The velocity contours are shown in FIGURE 22.

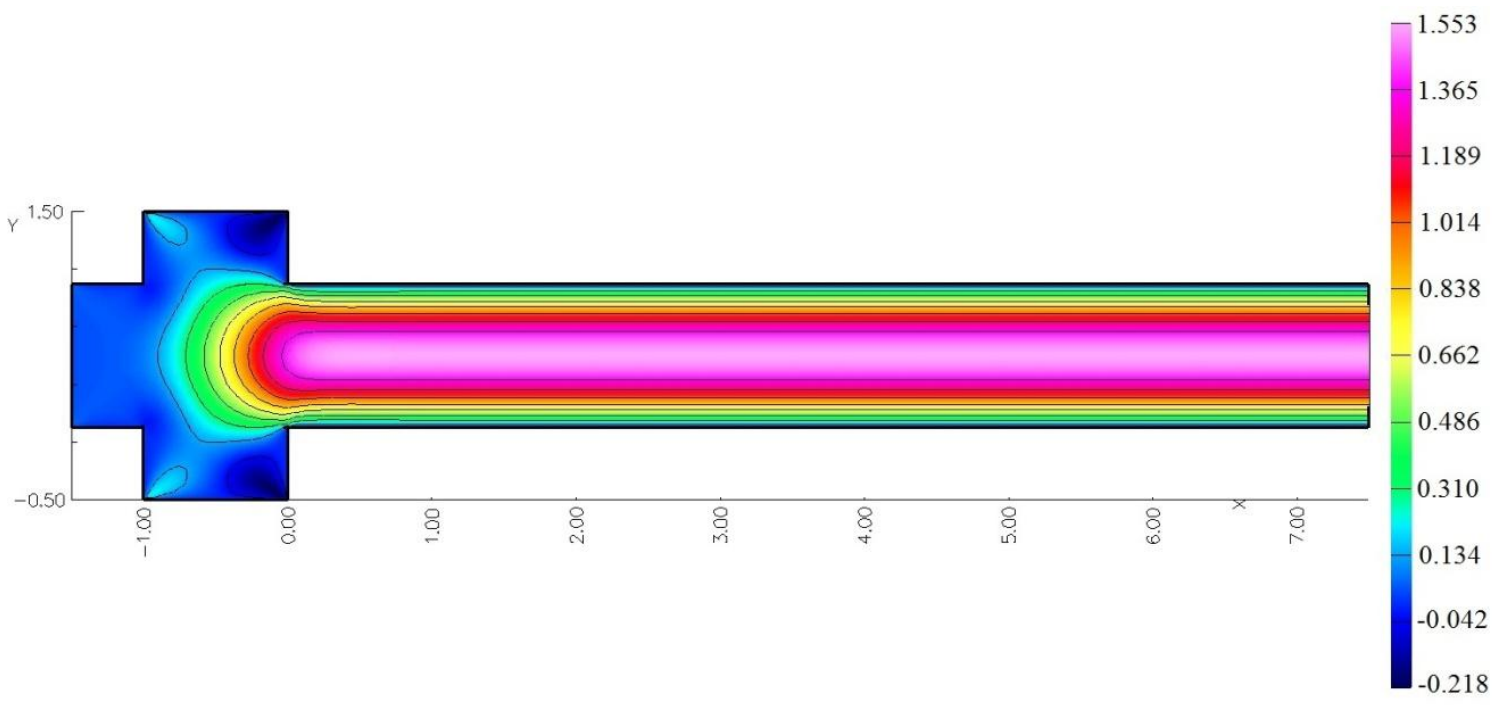

FIGURE 22 - Numerical solution for micro-channel in the u direction.

The streamwise velocity profile at a dimensionless position $x=7.0$ is compared with the analytical solution. The microcytometer numerical solution produced a dimensionless maximum velocity of 1.553 which is $3.4 \%$ higher than the analytical solution. A velocity profile comparison between the analytical, basic, and microcytometer is shown in FIGURE 23. 


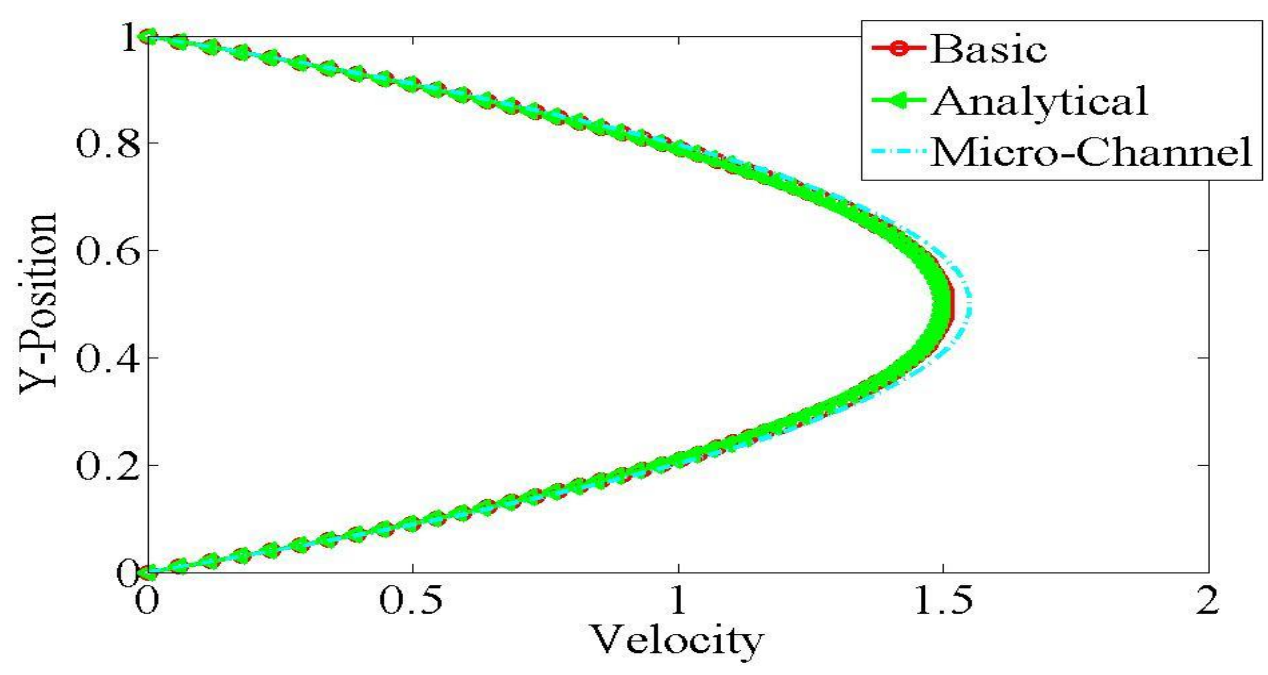

FIGURE 23 - Velocity profile comparison for Analytical, Basic, and Micro-Channel.

\section{$\underline{\text { C. Cell Motion in the Microcytometer }}$}

Cell separation within the channel needs to be considered because if the cells are too close together the Coulter Technique and the electronic system may miscount the actual number of cells. The electronic system used in Dr. Harnett's experiments will benefit from an increase in cell separation because a greater distance between the cells will allow the system to be more efficient in cell counting.

By adding two cells with a dimensionless diameter of .01 into the sample stream a representation of how each cell will react in fluid flow can be seen. Insertion of the cells into the microcytometer grid is done with the code shown in Appendix VIII. Running this code through Ogen, produces a grid shown in FIGURE 24. 


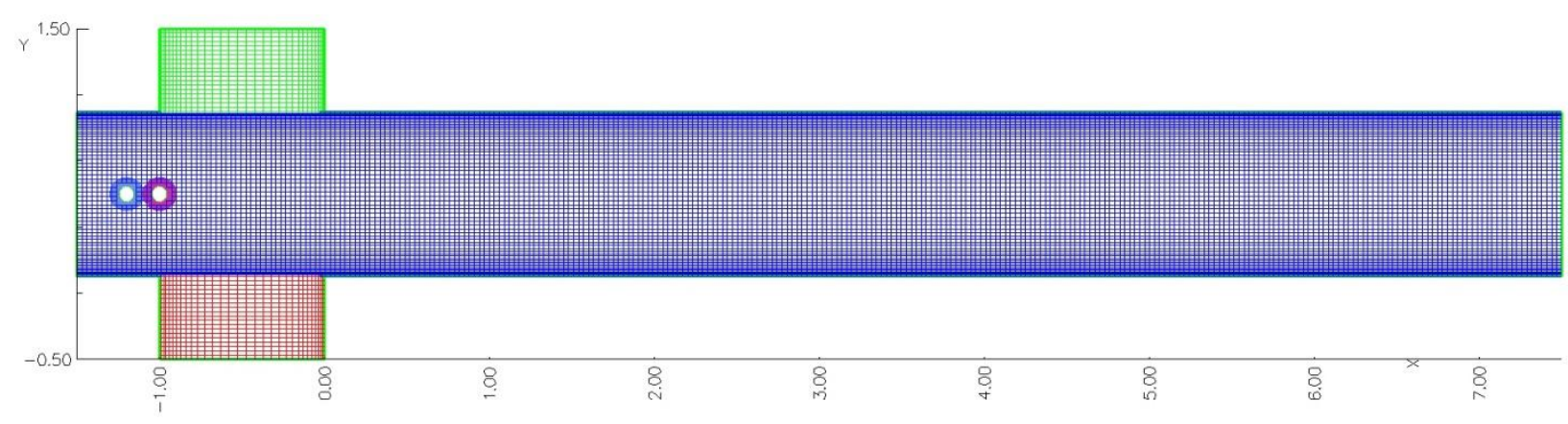

FIGURE 24 - Microcytometer grid with two cells inserted into the sample stream.

The option for moving grids is shown in the Cgins code of Appendix IX. The initial position of the cells is shown in FIGURE 25.

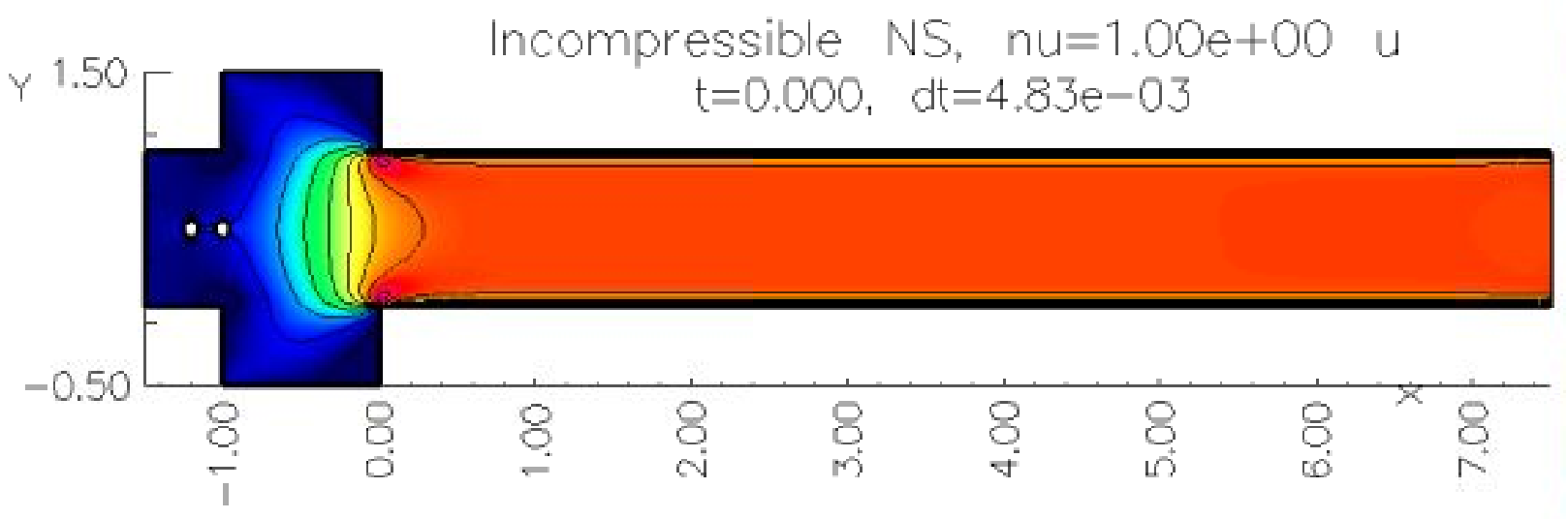

FIGURE 25 - Initial position of two cells on the channel when time is equal to zero.

As the cells begin to move under the hydrodynamic forces and approach the main channel they start to separate.

Separation of each passing cell can be controlled by the flow rate ratio. The flow rate ratio is defined as the inlet velocity of the sheath stream to the inlet velocity of the sample stream. A higher flow ratio will give greater separation between each cell. Keep in mind the flow ratio cannot be too large because the width of the focused stream starts to become smaller than the cell. Additionally, the flow ratio cannot be too small because the cells will be too close together, decreasing the counting efficiency. 
When the flow ratio is equal to 12 , the separation of the cells is around 3.25 and when the flow ratio is equal to 4.5 the cell separation is equal to 2.25 . This separation is displayed in FIGURE 26 and FIGURE 27.

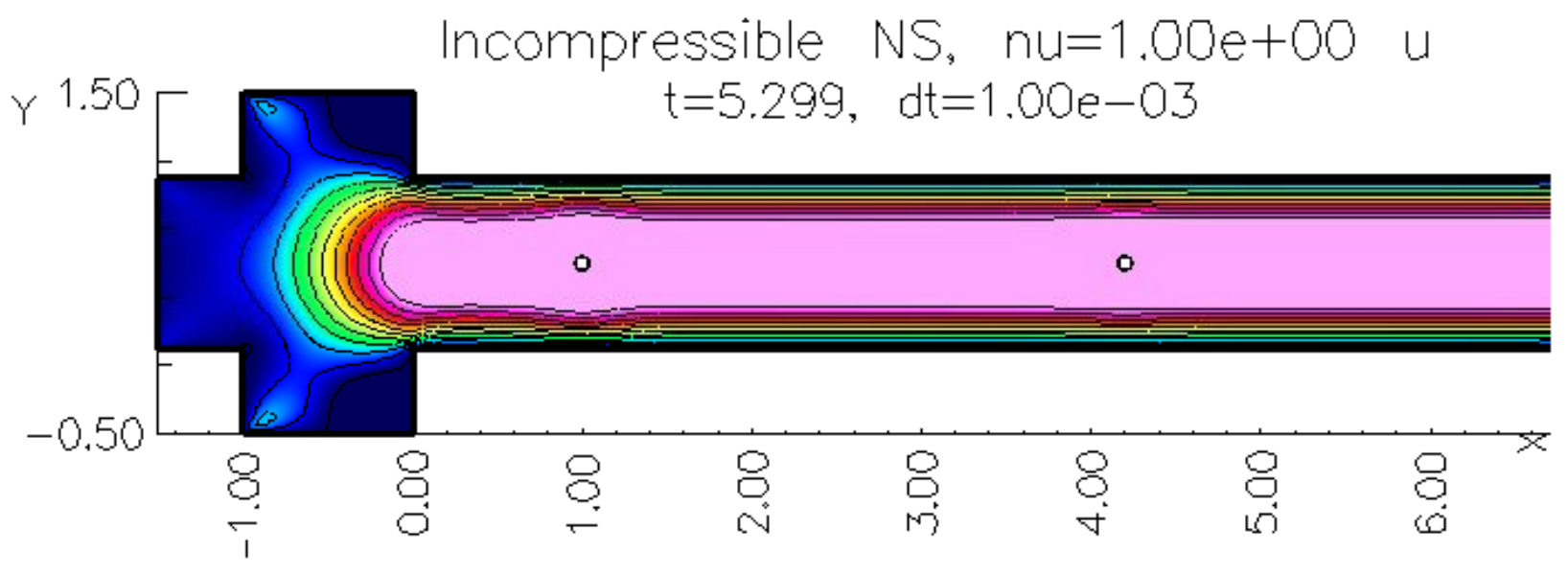

FIGURE 26 - Cell separation when the flow ratio is equal to 12.

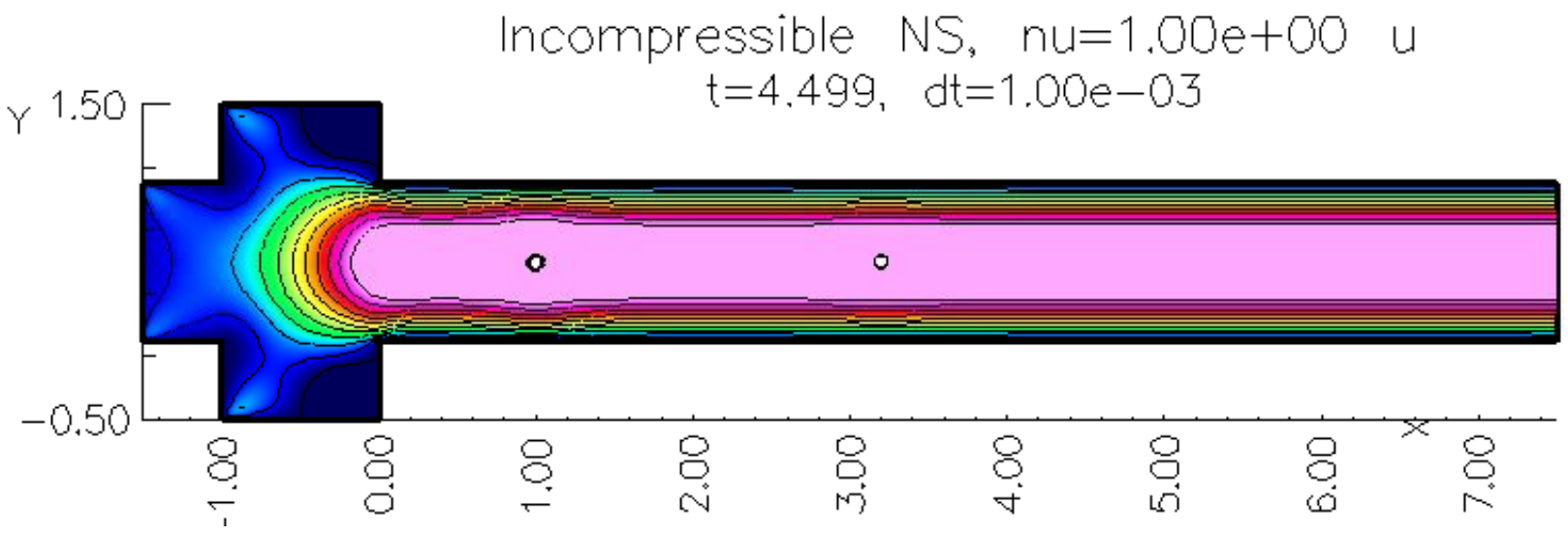

FIGURE 27 - Cell separation when the flow ratio is equal to 4.5.

The separation of cells is backed up by experimental results of (Lee, Chang, Huang, \& Yang, 2005). Lee states that as the sheath flow increases the separation of the cells will also increase. This increase in separation is caused by the shear force difference between the sheath and sample flows. Lee also states that symmetric hydrodynamic focusing is the best for cell/particle counters, and confirms that the 
focused stream should be about the same width as the cell/particle in order to help single out each cell (Lee, Chang, Huang, \& Yang, 2005). 


\section{MASS DIFFUSION WITHIN A MICROCYTOMETER}

This chapter investigates mass transfer why it's important to the microcytometer. Also, the effects of different Schmidt numbers on the diffusion rates are explored. Lastly, the effects of grid sensitivity on the diffusion solution and symmetric flow rate are examined to find the highest concentration level of the sample stream.

A high level of concentration is desirable because it can result in a larger and clearer resistance spike when a cell passes by the electrodes. By adjusting the flow ratio the sample stream concentration downstream is affected, and a clearer and more precise impedance signal can be obtained (Harnett, 2009).

Mass diffusion is defined as the passing of molecules of one substance containing a high concentration to a substance that contains a lower concentration. This occurs within gas mixtures, liquid solution, or a solid solution (Mills, 2001). Diffusion is also known as mass transfer, where in our case the mass transfer is occurring between the sample stream and the sheath streams.

A higher concentration liquid will have more molecule motion than a lower concentration liquid, the motion of molecules will continue until equilibrium is reached between the liquids (Mills, 2001). Fick's Laws of diffusion is the basis for all diffusion problems. Proposed in 1855 Fick's first law states a linear relationship exists between the 
local concentration gradient of a species and the rate of diffusion for its chemical species. The first law is analogous to Newton's law of viscosity, where a potential or driving force has a linear relationship to the flux (Mills, 2001).

Fick's Laws for diffusion of liquids keeps the convection and the density constant due to the fair temperature and pressure gradients: the resulting diffusion can be analyzed on a molar basis. The analysis on a molar basis gives Fick's Law can be expressed in the following two equations (Basmadjian, 2007).

$$
\text { Molar flow } N=-D A \frac{d C}{d x}(\text { moles } / \mathrm{s})
$$

and, also

$$
\text { Molar flux } N / A=-D \frac{d C}{d x}\left(\text { moles } / \mathrm{m}^{2} \mathrm{~s}\right)
$$

where $\mathrm{D}$ is the mass diffusivity, $\mathrm{A}$ is the area, and $\mathrm{C}$ is the concentration (Basmadjian, 2007).

Diffusion is not only affected by the mass flow rate but also by the laser source heating the cells. Forced diffusion from the electrodes can happen when an electrical field is imposed on an electrolyte. This type of diffusion can be very large when there is a strong electric field. Thermal diffusion, from the heat of the cell, happens when there is a large temperature gradient; this type of diffusion is also known as the Soret effect (Mills, 2001).

Thermal diffusion increases diffusion between the sample stream and the sheath stream. As the cell travels increased diffusion will allow more Ions to move into the 
sheath stream increasing its conductivity thus allowing an easier current path. Forced also increases diffusion between the sample and sheath stream by exciting the Ions in the sample stream (Harnett, 2009).

The Schmidt number is defined in EQUATION 9.

$$
S c=\frac{v}{D}
$$

Where $\mathrm{v}$ is the momentum diffusivity and $\mathrm{D}$ is the mass diffusivity. In experiments of Harnett, (Harnett, 2009) a dye with low mass diffusivity (FIGURE 28) is used, this leads to a high Schmidt number of 2000 (Scott, Sethu, \& Harnett, 2008).

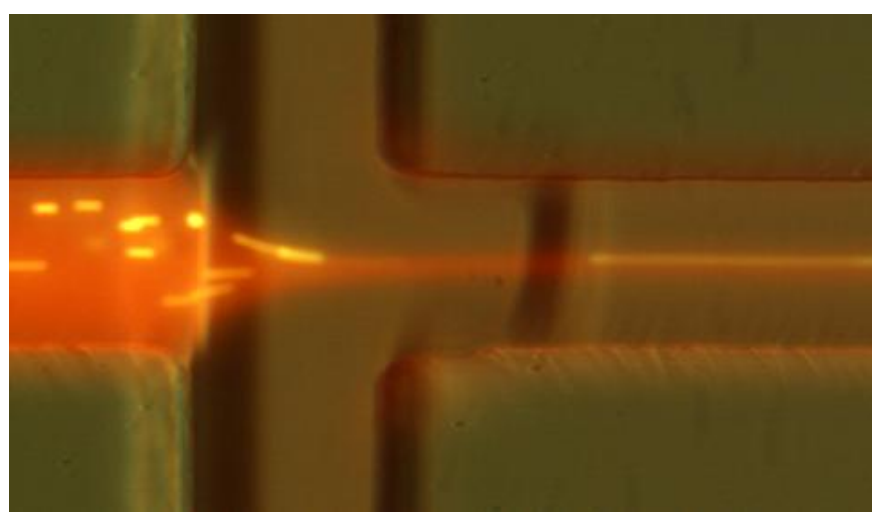

FIGURE 28 - Dye used for measuring the concentration in the sample stream.

Such a high Schmidt number makes it easier to observe the hydrodynamic focusing. A comparison of concentration between the low mass diffusivity dye $(\mathrm{Sc}=2000)$ and the high diffusivity ionic water $(\mathrm{Sc}=500)$ is shown in FIGURE 29. It is clear that at the low Schmidt number the sample flow is more spread out. 


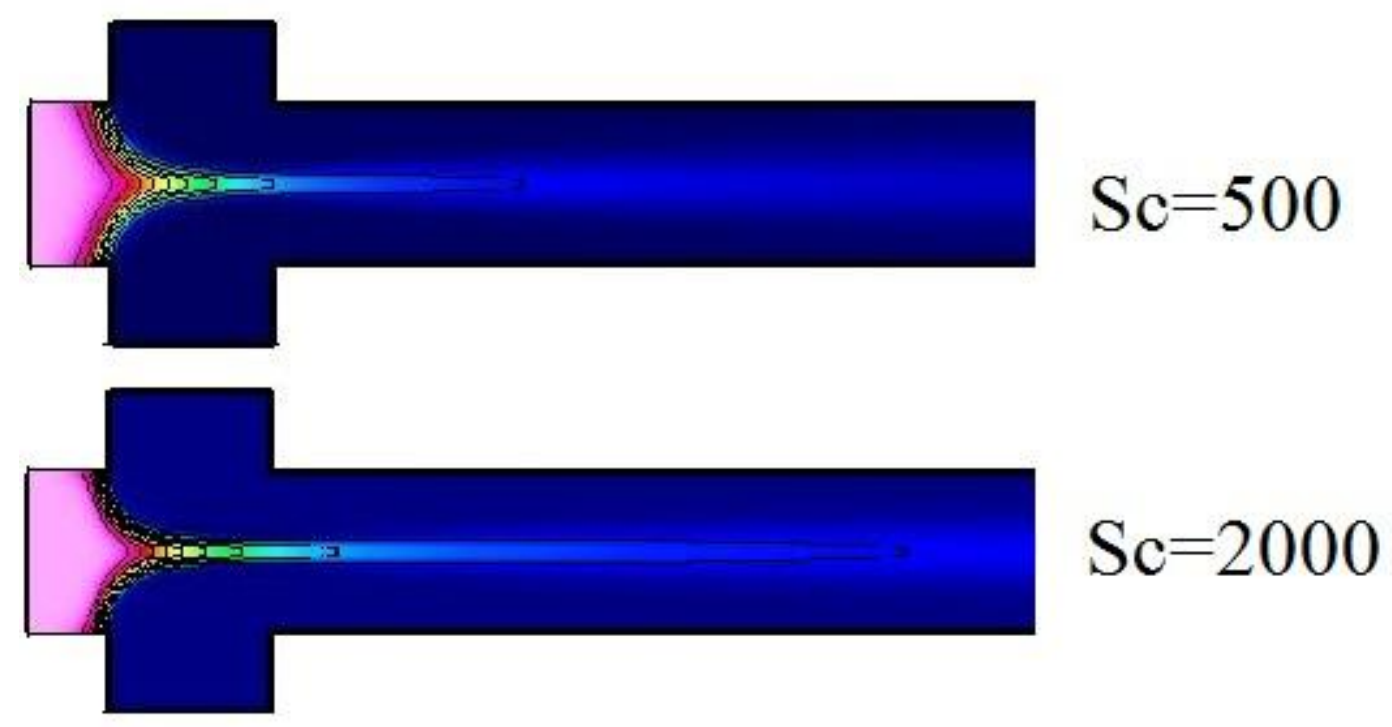

FIGURE 29 -Effect of Schmidt number on sample flow concentrations.

\section{$\underline{\text { A. Experimental Diffusion }}$}

Harnett et al. (Harnett, 2009) experimentally studied the concentration level in a microcytometer. In their work a customized MATLAB program evaluates recorded images (FIGURE 30) from a PC and turns them into a scatter plot (FIGURE 31). The program initially removes any noise within the picture with an adaptive noise-removal filter, in which the noise is assumed to have a Gaussian distribution. After noise removal is completed, each pixel is assigned an intensity level. This process is completed throughout the entire picture. 


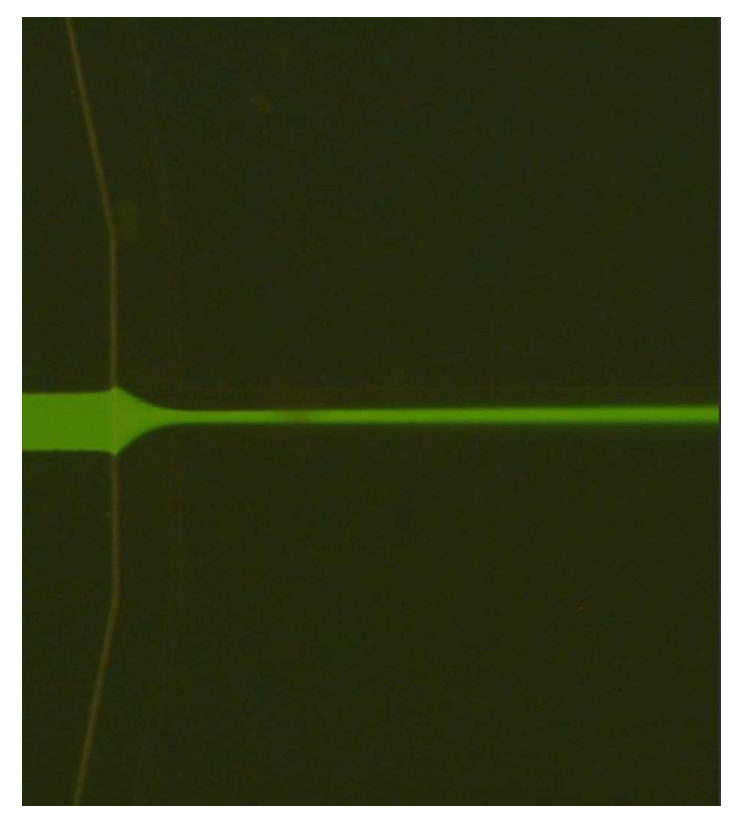

FIGURE 30 - Experimental hydrodynamic focusing of dye.

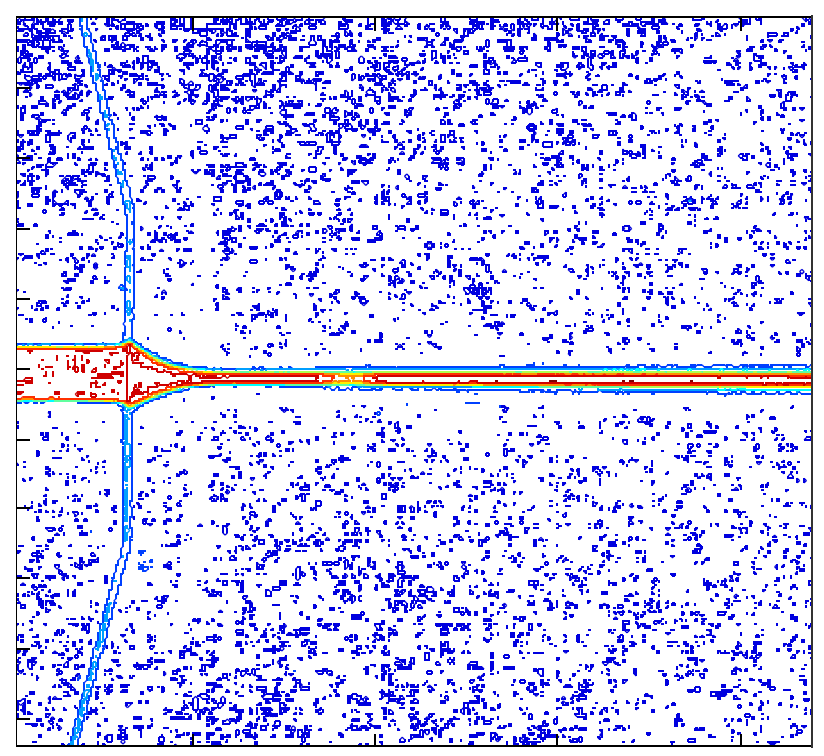

FIGURE 31 - MATLAB results of concentration levels.

In FIGURE 31 the red has a concentration level equal to one and dark blue has a concentration level equal to zero.

B. Diffusion Grid Sensitivity 
As with finding the velocity distribution in a channel, grid sensitivity must be performed for the mass diffusion between the sample and sheath streams. Changing $\mathrm{x}-$ direction and y-direction resolution will affect the diffusion solution. A comparison between Wu \& Nguyen (2005) normalized results, Harnett et. al. Harnett (2009) MATLab results, and the numerical results of the microcytometer can be completed. Cgins command file to solve for diffusion is shown in Appendix X.

Increasing the $\mathrm{x}$-direction grid lines changes the diffusion level by a small amount. This can be seen in FIGURE 32.

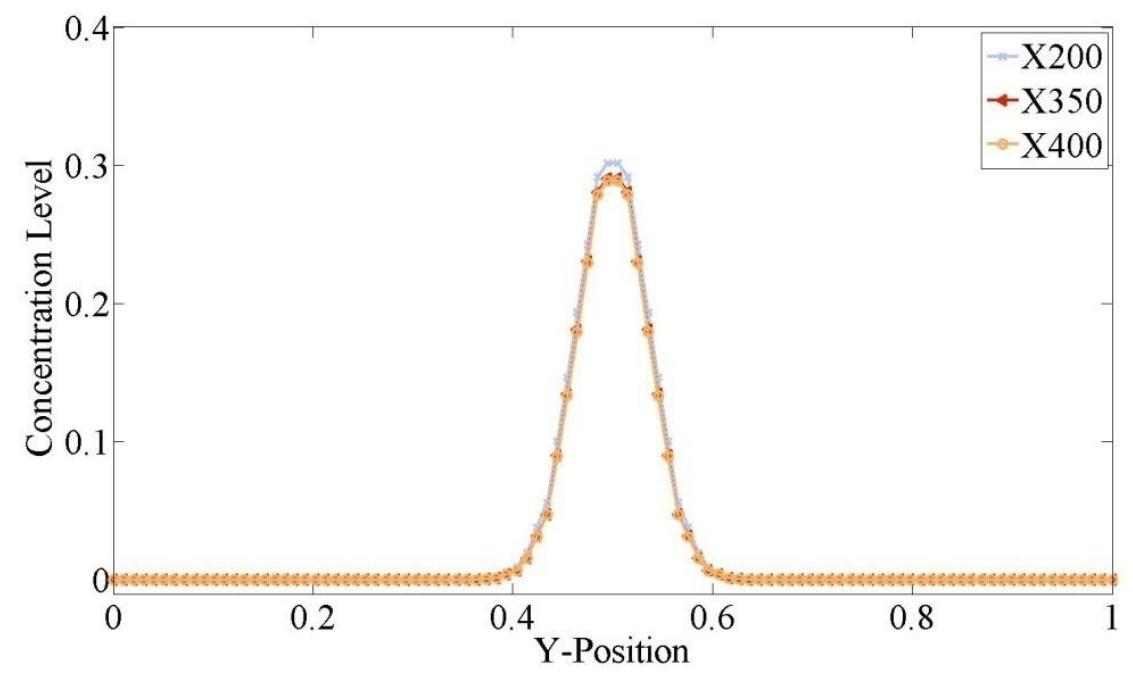

FIGURE 32 - Concentration level, x-position of .5, along the width of the channel.

A closer view of FIGURE 32 shows the concentration level converging, shown in FIGURE 33. 


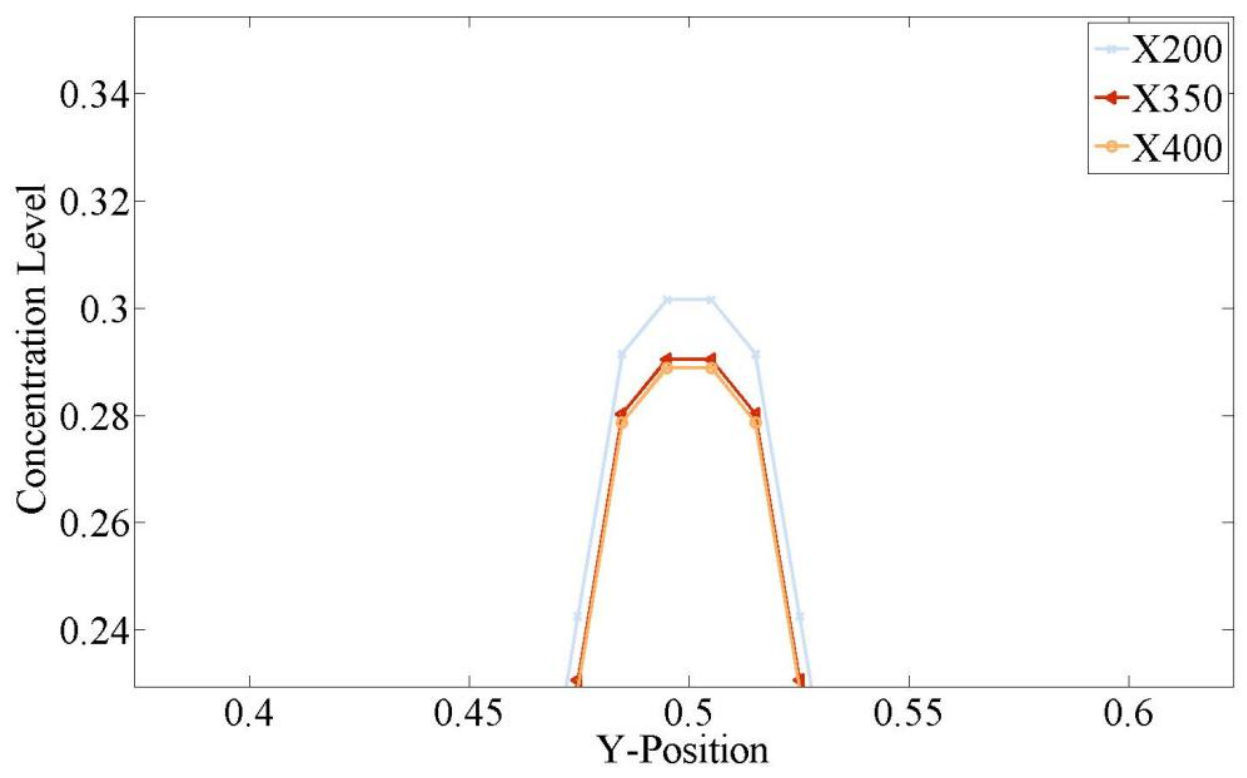

FIGURE 33 - Closer view of FIGURE 32.

From FIGURE 33 the grid lines converge on to a concentration level when the $\mathrm{x}$ direction grid lines are near 350. This also shows that the $\mathrm{x}$-directional grid lines have hardly any effect on the diffusion solution.

Evaluation of TABLE III will conclude the concentration level is more sensitive to the number of grid points in the y-direction.

TABLE III

EFFECT OF DIFFUSION BY INCREASING Y-DIRECTIONAL GRID LINES

\begin{tabular}{|c|c|c|c|c|}
\hline Case & 1 & 2 & 3 & 4 \\
\hline Y-Direction & 64 & 200 & 64 & 128 \\
\hline X-Direction & 200 & 200 & 450 & 450 \\
\hline Concentration Level & 0.3015 & 0.3767 & 0.2871 & 0.3512 \\
\hline Time(hrs) & 30 & 99 & 48 & 60 \\
\hline
\end{tabular}

Results from TABLE III show that by increasing the y-direction grid lines the concentration level is dramatically changed, even when there is an increase in $\mathrm{x}$-direction 
grid lines. However the cpu time to solve increased Y-direction grid lines of the microcytometer grid is a bigger concern than the $17 \%$ difference between concentration levels. Thus a grid using $350 \mathrm{x}$-direction grid lines and $64 \mathrm{y}$-direction grid lines will be used because this grid will show a reasonable result without using extra processing time.

An experimental normalized diffusion solution of Wu \& Nguyen (2005) is plotted in FIGURE 34. The decay of the maximum concentration is clearly shown.

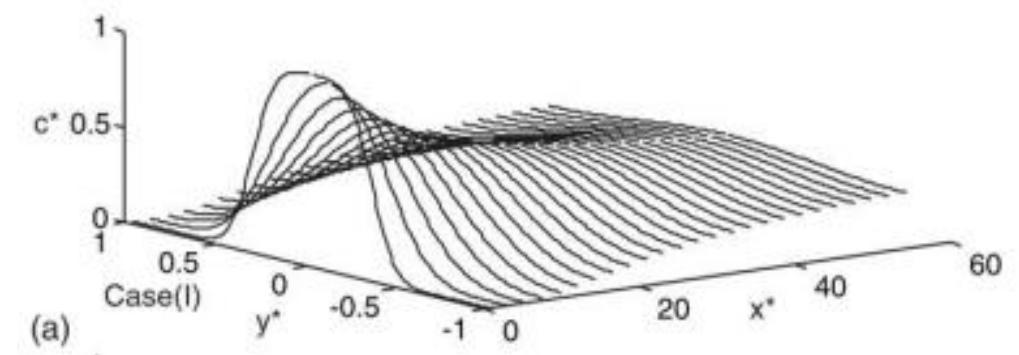

FIGURE 34 - Normalized diffusion along a channel.

$\mathrm{x}^{*}=\mathrm{x} / \mathrm{W}, \mathrm{y}^{*}=\mathrm{y} / \mathrm{W}, \mathrm{c}^{*}=\mathrm{c} / \mathrm{Co}$, and $\mathrm{Co}$ is the initial concentration level. These normalized results can be compared with the numerical results produced from the microcytometer grid, shown in FIGURE 35. 

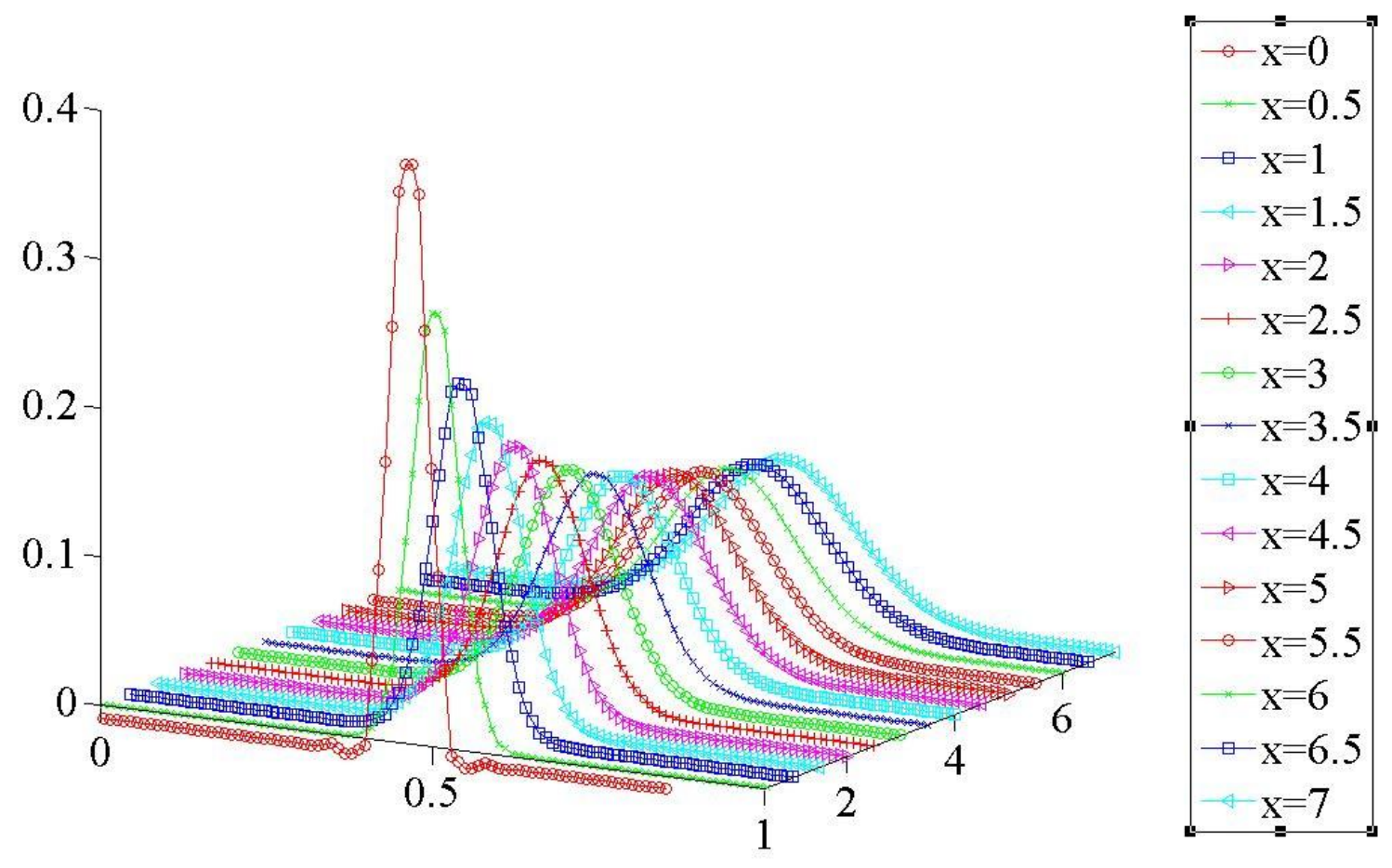

FIGURE 35 - Diffusion down the length of the channel.

When comparing FIGURE 34 and FIGURE 35, our numerical solution illustrates a similar trend with the normalized results from Wu \& Nguyen (2005). When $\mathrm{x}$ is increased along the channel the concentration of the fluid starts out high and skinny then decreases and fans out. This comparison tells us that the grid used for a numerical diffusion solution is giving us a close proximity of the normalized solution.

A comparison between the numerical diffusion solution (FIGURE 36) and (Harnett, 2009) MATLab results from FIGURE 31, shows the microcytometer grid is capturing the hydrodynamic focusing, for the rest of the channel diffusion is happening too quickly. This is due to the insufficient grid lines in the center of the channel. 


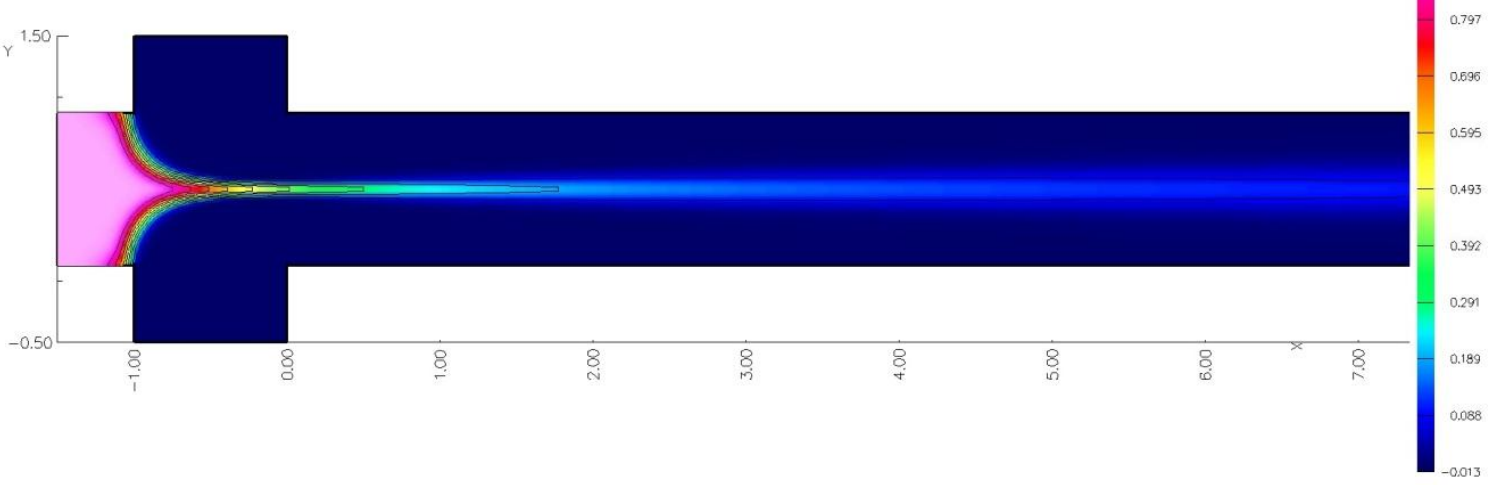

FIGURE 36 - Numerical result for diffusion.

\section{Effects of Different Asymmetric and Symmetrical Flow Rates}

In this section different symmetrical flow rates between the sample and sheath streams are investigated to see the affect on the concentration level of the samples stream. Ultimately the best ratio will have the highest concentration level near the electrodes and a focused width roughly the same width as the cell diameter. Also, how different asymmetric flow rates will affect the position of the focused stream was investigated. TABLE IV show flow rates to be analyzed.

TABLE IV

FLOW RATES USED IN DIFFUSION ANALYSIS

\begin{tabular}{|c|c|c|}
\hline Sample Stream & Sheath Streams & Flow Ratio \\
\hline 0.010 & 0.495 & 49.500 \\
\hline 0.020 & 0.490 & 24.500 \\
\hline 0.030 & 0.485 & 16.167 \\
\hline 0.040 & 0.480 & 12.000 \\
\hline 0.050 & 0.475 & 9.500 \\
\hline 0.060 & 0.470 & 7.833 \\
\hline 0.070 & 0.465 & 6.643 \\
\hline
\end{tabular}




\begin{tabular}{|l|l|l|}
0.080 & 0.460 & 5.750 \\
\hline 0.090 & 0.455 & 5.056 \\
\hline 0.100 & 0.450 & 4.500 \\
\hline 0.200 & 0.400 & 2.000 \\
\hline 0.300 & 0.350 & 1.167 \\
\hline 0.400 & 0.300 & 0.750 \\
\hline 0.500 & 0.250 & 0.500 \\
\hline
\end{tabular}

The flow ratio can be calculated using EQUATION 10.

$$
\text { FlowRatio }=\frac{Q_{\text {sheathstram }}}{Q_{\text {samplestram }}}
$$

FIGURE 37 shows the focused stream width increasing as the flow ratio decreases.

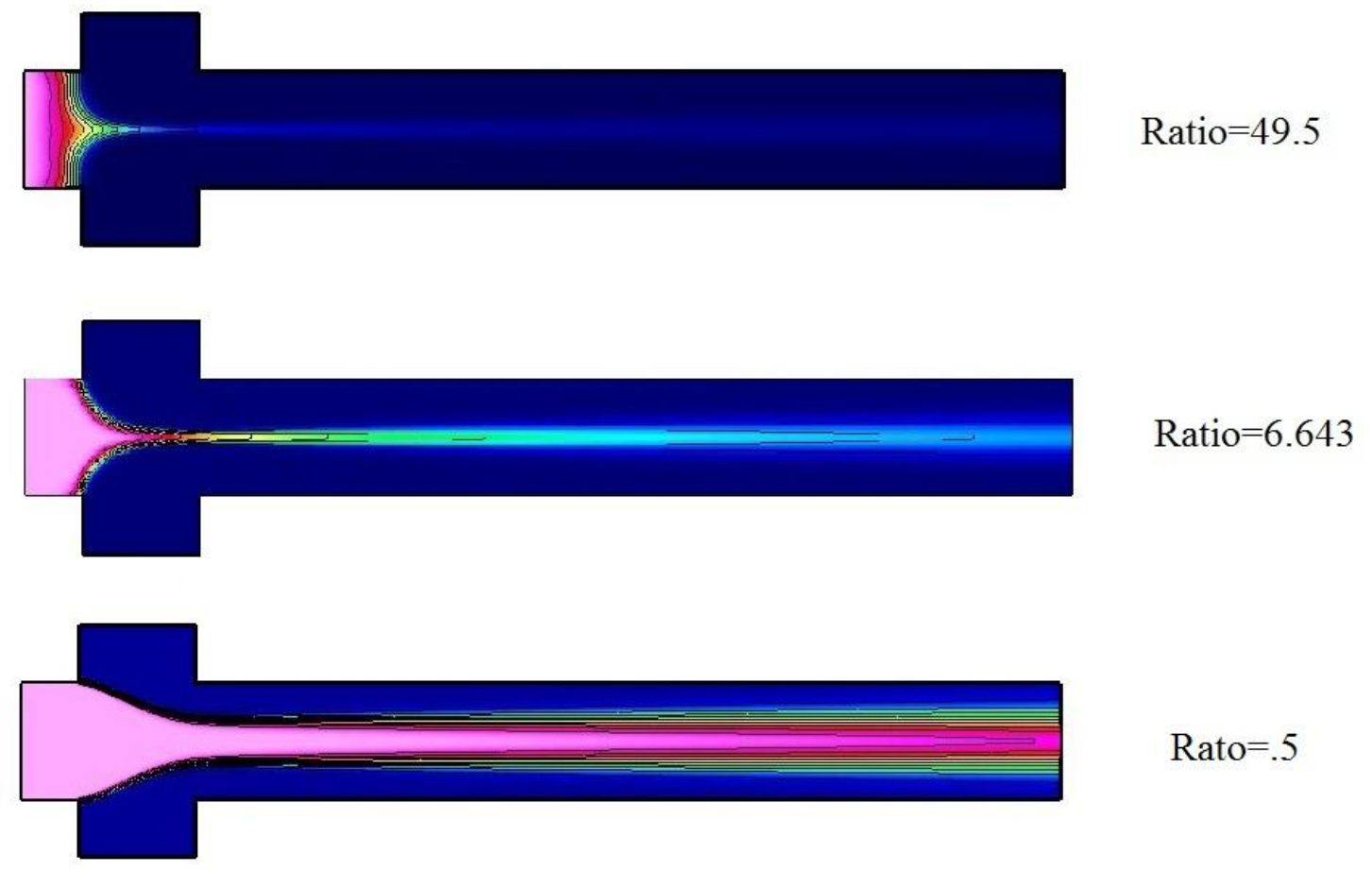

FIGURE 37 - Increased focused stream width as the flow ratio decreases. 
FIGURE 38 compares the concentration levels at $\mathrm{x}=0.5$ using the flow rates of TABLE IV.

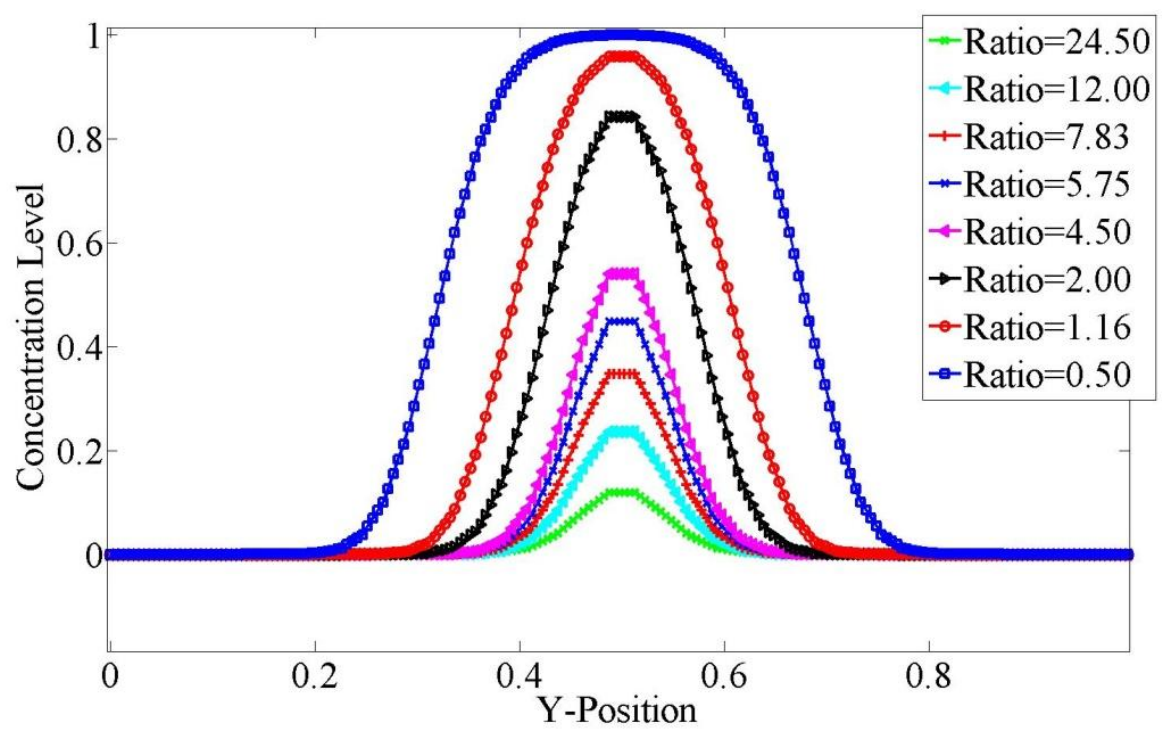

FIGURE 38 - Concentration level comparison between different flow rates $\mathrm{x}=0.5$.

Experiments have shown that the clearest impedance signal occurs when the focused stream roughly the same as the cell width (Scott, Sethu, \& Harnett, 2008). Applying this parameter a ratio can be chosen from FIGURE 38. Chosen ratios from FIGURE 38 that keep the focused stream roughly the same as the cell are in the range between 6.643 and 5.056 .

Asymmetric flow rates allow the focused stream to move within the channel. This can be useful when trying to move cells to a certain point within the channel. For the numerical simulation a flow ratio of 6.643 was used because this will keep the focused width the same width as the cell. The changes between the upper and lower sheath streams can be seen in TABLE V. 
TABLE V

ASYMMETRIC FLOW RATIOS BETWEEN UPPER AND LOWER SHEATH STREAMS

\begin{tabular}{|c|c|c|}
\hline Lower Sheath & Upper Sheath & Flow Ratio \\
\hline 0.475 & 0.455 & 0.958 \\
\hline 0.485 & 0.445 & 1.090 \\
\hline 0.495 & 0.435 & 1.138 \\
\hline 0.505 & 0.425 & 1.188 \\
\hline 0.515 & 0.415 & 1.241 \\
\hline 0.555 & 0.375 & 1.480 \\
\hline 0.595 & 0.335 & 1.776 \\
\hline 0.635 & 0.295 & 2.153 \\
\hline 0.675 & 0.255 & 2.647 \\
\hline 0.715 & 0.215 & 3.326 \\
\hline
\end{tabular}

TABLE V numerical simulations are shown in FIGURE 39.

Where the asymmetric flow ratio is calculated using EQUATION 11.

$$
\text { FlowRatio }=\frac{Q_{\text {uppershealh }}}{Q_{\text {lowersheah }}}
$$




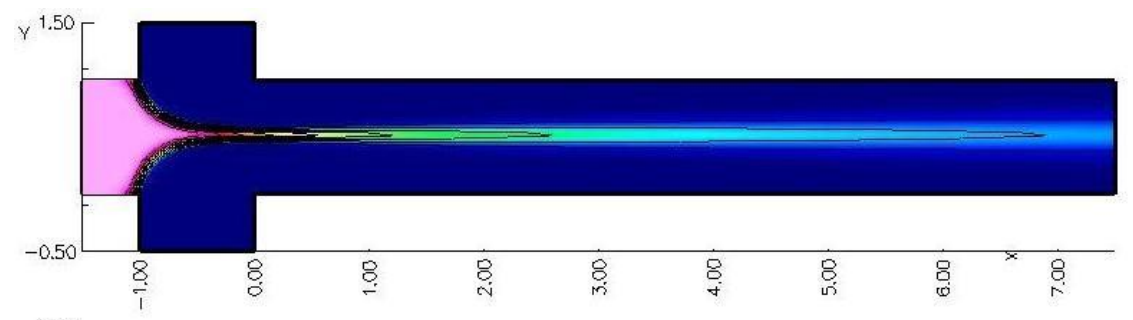

Flow Ratio $=1.14$

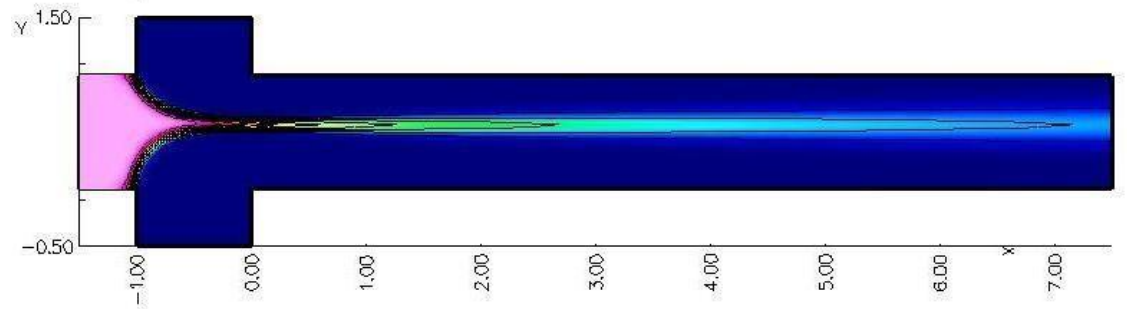

Flow Ratio $=1.48$

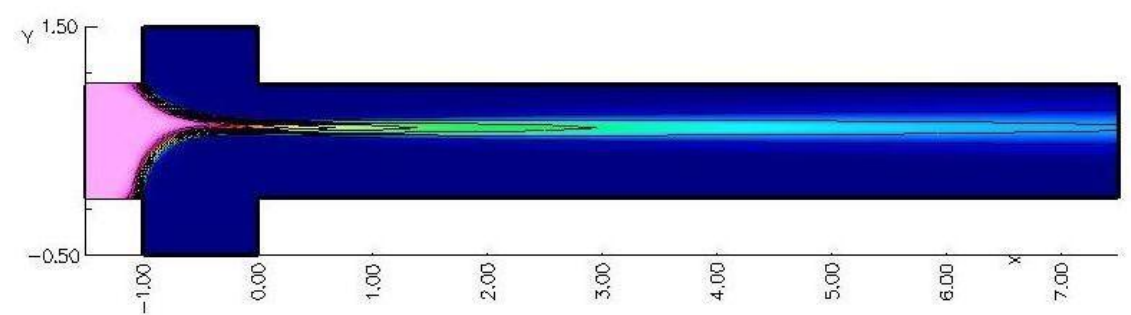

Flow Ratio $=2.15$

FIGURE 39 - Asymmetric flow comparison of different upper and lower sheath stream ratios.

The numerical results of the position of the focused stream can be compared with analytical results from (Lee, Chang, Huang, \& Yang, 2005) shown in FIGURE 40. 


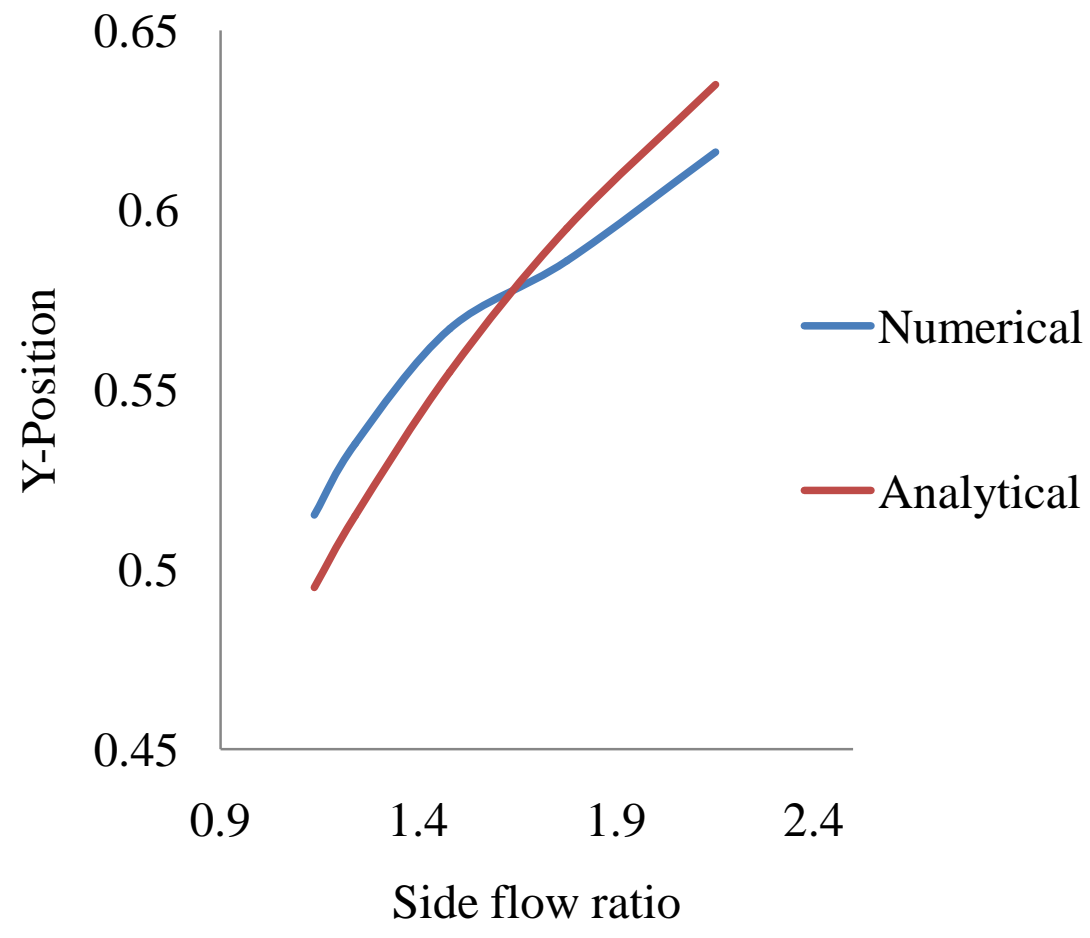

FIGURE 40 - Asymmetric flow position comparison between numerical and analytical.

Comparing these two solutions show a $2.88 \%$ difference between numerical and analytical results. 


\section{THREE DIMENSIONAL MICROCYTOMETER}

Here a three dimensional grid will show different viscous aspects of fluid flow and diffusion within the channel. A disadvantage from going from two dimensions to three dimensions is that a third dimension adds more grid lines, extra grid lines intern means that there will be an increased processing time.

\section{A. Three Dimensional Fluid Flow and Diffusion}

The only difference between creating and solving grids in three dimensions in Overture is the creation of boxes instead of rectangles. The boundary conditions applied are the same as two dimensions. Code from Appendix XI and Appendix XII can be used to create and numerically solve a three dimensional microcytometer. A three dimensional numerical velocity plot is shown in FIGURE 41. 


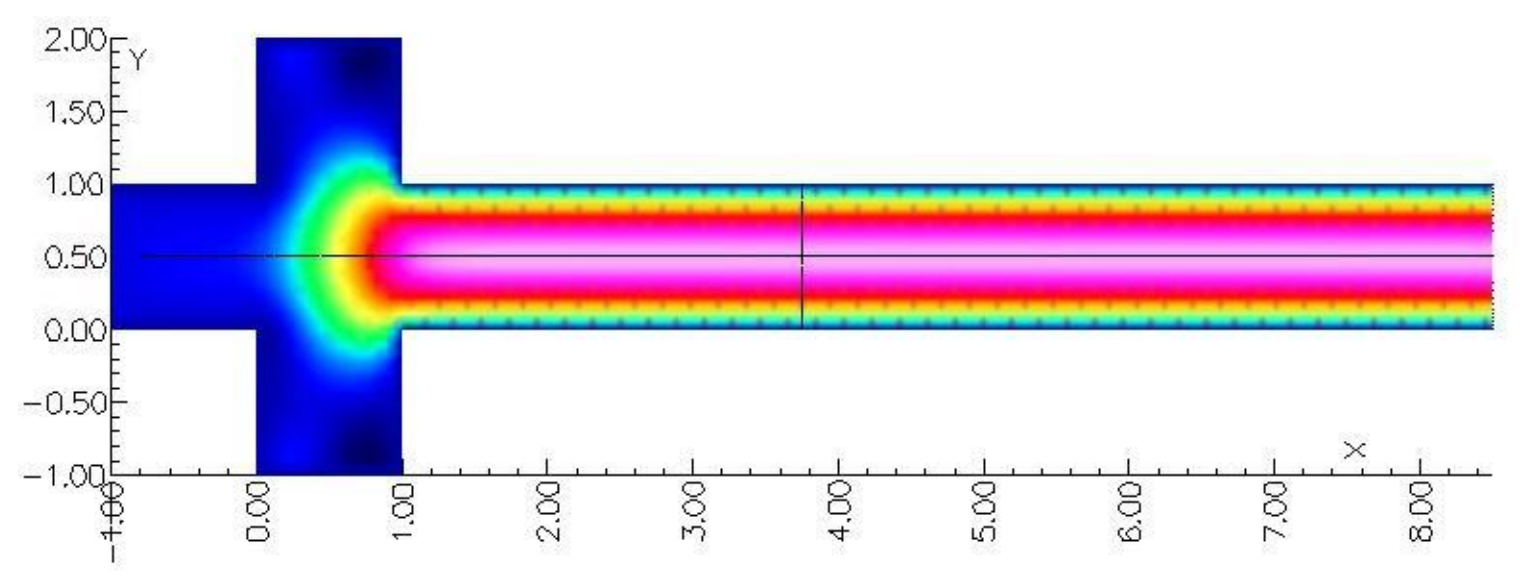

FIGURE 41 - Three dimensional numerical velocity solution.

The transition from two dimensions to three dimensions allows a solution to be seen across the entire $\mathrm{Y}-\mathrm{Z}$ plane of the channel. This is represented by a bullet like profile, where the velocity increases as you move towards the center of the channel, shown in FIGURE 42.

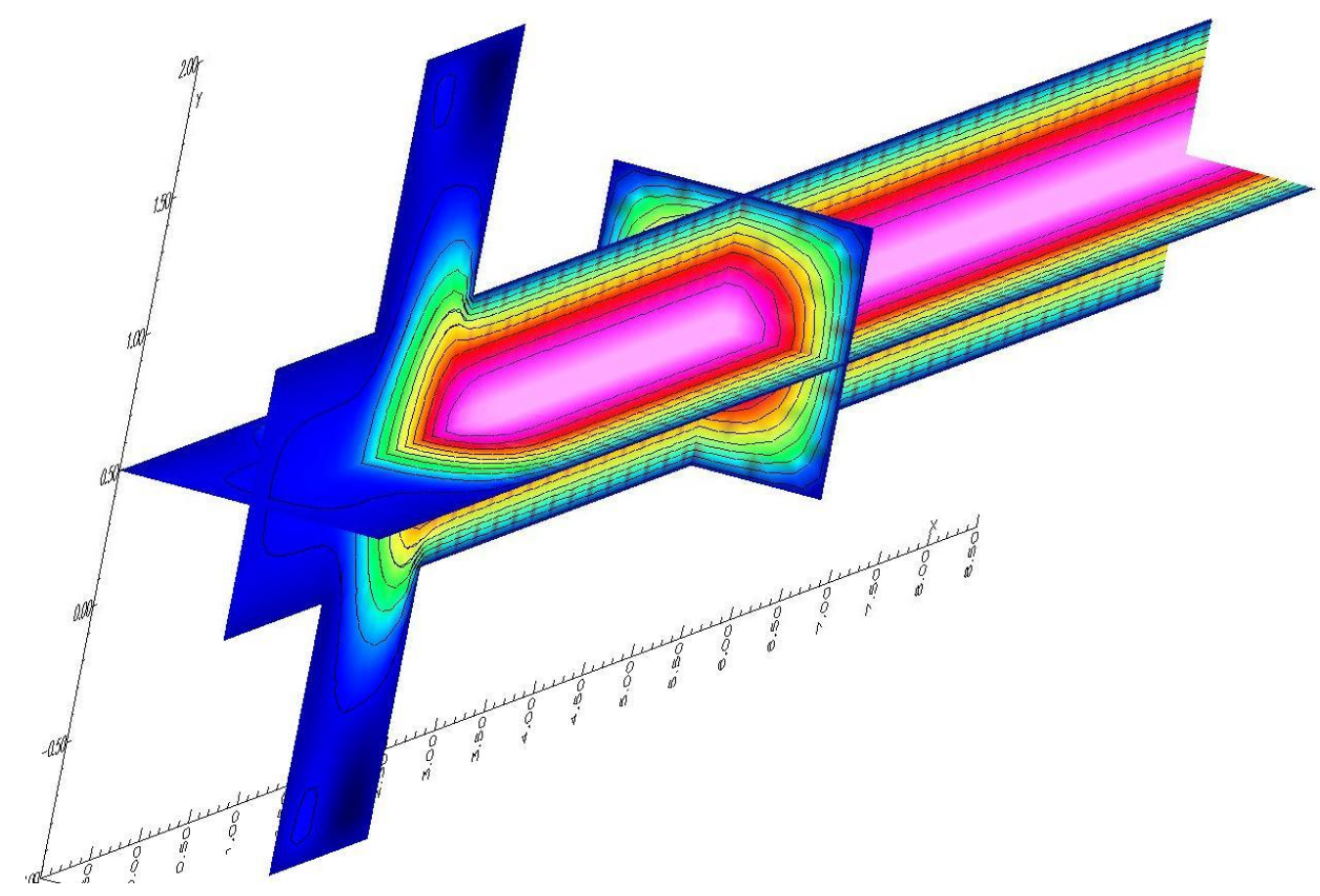

FIGURE 42 - Three dimensional numerical velocity solution showing "bullet" profile. 
For diffusion within the channel, one aspect that can be seen in three dimensions and not in two dimensions is the viscous effects that are created near the walls of the channel. Experimental results have indicated an hour glass effect when taking the cross section of the microcytometer at any given x position (Harnett, 2009).

The numerical solution and hour glass viscous effects of the diffusion are shown in FIGURE 43.

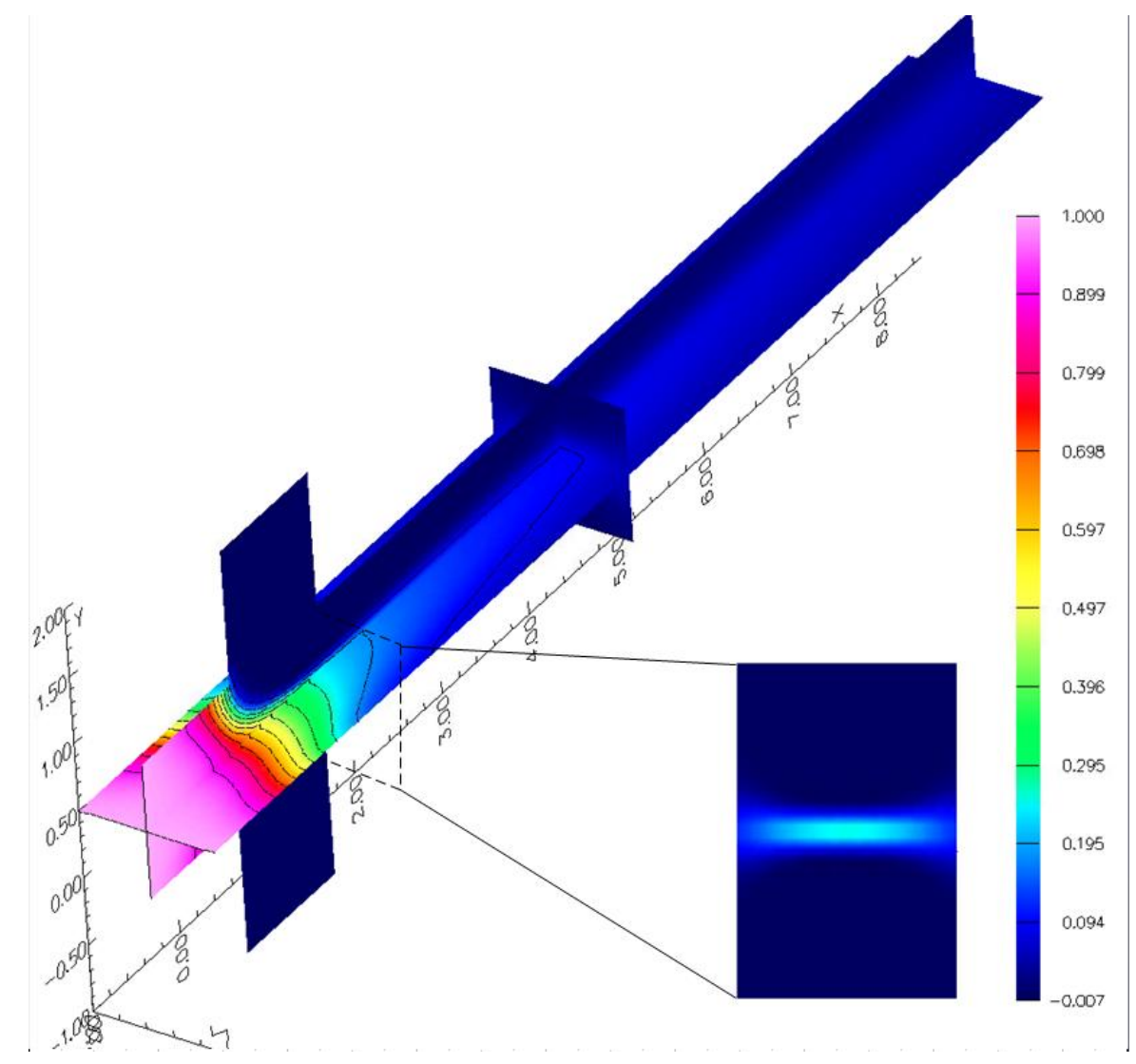

FIGURE 43 - Diffusion of the three dimensional channel with a cross section showing viscous near the walls.

This hour glass shape shows that the viscous effects of the fluid are being captured in the numerical simulation. 


\section{CONCLUSIONS}

After many hours of grid failures and grid refinement, a grid of $64 \mathrm{y}$-direction grid lines and $350 \mathrm{x}$-direction grid lines proved to match up well with the fluid flow analytical solution. The same grid also showed that the maximum concentration levels are optimized when the flow ratio is between 6.643 and 5.056. This ratio followed the condition where that the focused stream needed to be roughly the same width as the cell. The three dimensional numerical solution showed viscous effects of the fluid on the walls are being captured by an hour glass shape. 


\section{RECOMMENDATIONS}

1. Move the location of the electrodes closer to the front of the exit channel. This will allow the for the highest concentration level of the sample stream at the electrodes.

2. Move the sample stream closer to the electrodes, this will allow the heated cell and electrodes to have a small amount of sheath stream to diffuse into. 


\section{FUTURE WORK}

Additional grid refinement will be needed for diffusion of the channel. Even though the microcytometer grid is capturing the diffusion fairly well, it is still not capturing it enough.

More grid lines in the y-direction will help capture the diffusion better; however this will take more cpu time to solve. Stretching towards the middle of the channel cannot be done because it will take away from the velocity solution. FIGURE 44 shows a possible grid solution for capturing the diffusion.

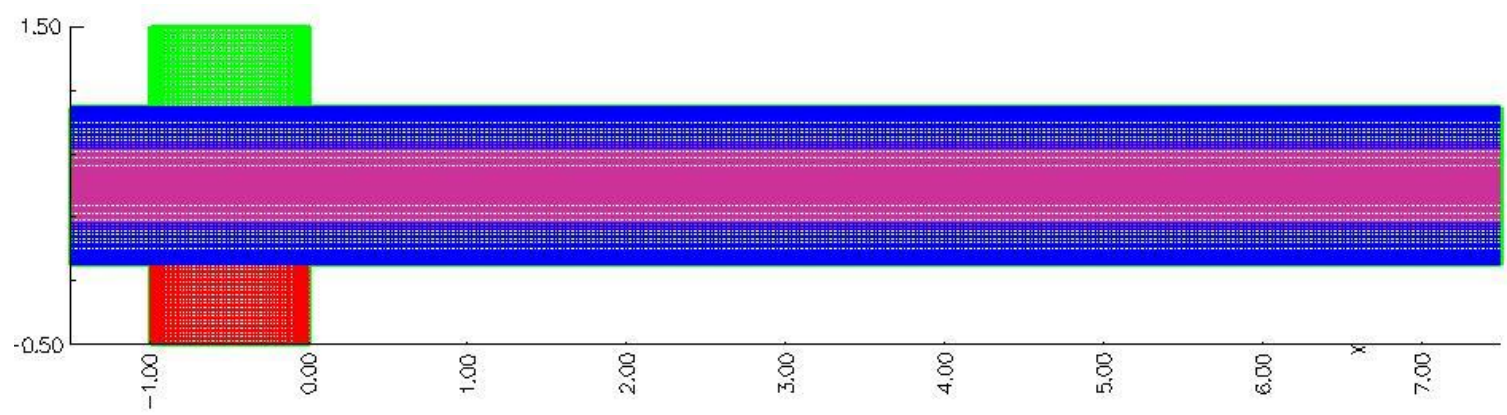

FIGURE 44 - Added focused grid.

This grid configuration uses four grids instead of three. The fourth grid can be used to capture the diffusion and not increase processing time. This additional grid can also be moved up or down in the when asymmetric focusing is wanted. 
Heat transfer from the heat source, continuation of the three dimensional grid and evaluation of the electrical field will need to be done in order to finish out the rest of the CFD microcytometer analysis. 


\section{APPENDIX I}

*Creating the channel create mappings

rectangle

set corners

0.150 .1

lines

35032

exit

*Stretching the grid

stretch coordinates

transform which mapping?

square

stretch

specify stretching along axis $=1$

stretching type

hyperbolic tangent

hyperbolic tangent parameters

.5 .0311 .03 .5

exit

exit

ccs

exit boundary conditions

1111

mappingName

ccs

exit

exit

*Generating the Grid

generate an overlapping grid

ccs

done

change parameters

ghost points

all

222222

exit

compute overlap

exit

*Saving the Grid

save an overlapping grid

ccs.hdf 


\section{APPENDIX II}

* cgins command file for flow in a channel.

* specify the overlapping grid to use: ccs.hdf

* Specify the equations we solve: incompressible Navier Stokes exit

* Next specify the file to save the results in.

show file options

compressed

open

ccs.show

frequency to flush

3

exit

* display parameters

turn off twilight zone

* choose implicit time stepping:

implicit

* but integrate the square explicitly: choose grids for implicit

all=implicit

ccs=explicit done

final time 15 .

times to plot .5

plot and always wait

pde parameters

nu

.01

done

boundary conditions

all=noSlipWall

$\operatorname{ccs}(0,0)=$ inflow With VelocityGiven, uniform $(p=1 ., u=1$.

$\operatorname{ccs}(1,0)=$ outflow,

$\operatorname{ccs}(0,1)=$ noSlipWall

$\operatorname{ccs}(1,1)=$ noSlipWall

done

initial conditions

uniform flow

$\mathrm{p}=1$., $\mathrm{u}=1$.

exit

project initial conditions

continue

movie mode

finish 


\section{APPENDIX III}

get a solution

*enter a solution \#

*ie 23

contour

plot:u

line plots

specify lines

*specify the number of velocity lines and point per line $*_{\text {ie }} 5,21$

add u_line_1

*continue to enter the previous line until specified number is met

save results to a matlab file

exit

exit

exit

exit 


\section{APPENDIX IV}

*Creating the channel create mappings

rectangle

set corners

0.150 .1

*create lines [h v]

lines

30032

exit

stretch coordinates

transform which mapping?

square

stretch

specify stretching along axis=1

stretching type

hyperbolic tangent

hyperbolic tangent parameters

.5 .0311 .03 .5

exit exit

exit

stretch coordinates

transform which mapping?

stretched-square

stretch

specify stretching along axis $=0$

stretching type

exponential

exponential parameters

$0, .75,1.0,6.0, .9$

exit

exit

boundary conditions

1111

mappingName

ccs

exit

exit 


\section{APPENDIX V}

Creating the channel
create mappings
rectangle
set corners
6.5150 .1
mappingName
ccs 2
$[\mathrm{~h}$ v]
lines
7532
exit
stretch coordinates
transform which mapping?
ccs 2
stretch
specify stretching along axis=1
stretching type
hyperbolic tangent
hyperbolic tangent parameters
.5 .0311 .03 .5
exit
exit
mappingName
ccs
boundary conditions
0111
exit
rectangle
set corners
0.6 .50 .1
mappingName
ec2
*[h v]
lines

37532

exit

stretch coordinates

transform which mapping?

ec2

stretch

specify stretching along axis $=1$ stretching type

hyperbolic tangent

hyperbolic tangent parameters

.5 .0311 .03 .5

exit

exit

mappingName

ec

boundary conditions

1011

exit

exit

generate an overlapping grid

ces

ec

done

change parameters

ghost points

all

222222

exit

compute overlap

exit

save an overlapping grid

ccs.hdf

ccs

exit 


\section{APPENDIX VI}

*Create a channel that has three inlet channels

create mappings

* Making of the upper sheath stream rectangle

set corners

0. -11.51

lines

6432

exit

stretch coordinates

transform which mapping?

square

stretch

specify stretching along axis $=0$

stretching type

hyperbolic tangent

hyperbolic tangent parameters .5 .0311 .03 .5

exit

exit

boundary conditions

1110

share

8867

mappingName

uppersheath

exit

delete a mapping

square

*making of the lower sheath stream

rectangle

set corners

$0-10,-.5$

lines

6432

exit

stretch coordinates

transform which mapping?

square

stretch

specify stretching along axis $=0$

stretching type hyperbolic tangent

hyperbolic tangent parameters .5 .0311 .03 .5

exit

exit

boundary conditions

1101

share

12121513

mappingName

lowersheath

exit

delete a mapping

square

*Making the sample stream

rectangle set corners

$-1.57 .50 .1$

lines

35064

exit

stretch coordinates

transform which mapping?

square

stretch

specify stretching along axis $=1$

stretching type

hyperbolic tangent

hyperbolic tangent parameters .5 .0311 .03 .5

exit

exit

boundary conditions

1111

share

2017128

mappingName

samplestream

exit

delete a mapping

square

* now look at the mappings

view mappings 
uppersheath

lowersheath

samplestream

colour boundaries by share value

*pause

erase and exit

exit

generate an overlapping grid

* put the nonconforming grid first to be

a lower

* priority than the back-ground samplestream

uppersheath

lowersheath

done

change parameters

prevent hole cutting

lowersheath

samplestream

uppersheath

samplestream

samplestream lowersheath

samplestream

uppersheath

done

ghost points

all

222222

exit

* display intermediate

* set debug parameter

* 31

compute overlap

change parameters

interpolation type

exit

exit

*

save an overlapping grid mci.hdf

mci

exit 


\section{APPENDIX VII}

\# specify the overlapping grid to use:

mcih350.hdf

\# Specify the equations we solve:

incompressible Navier Stokes

passive scalar advection

exit

\#

\$ts

\$implicitVariation

*

* lian showfile

show file options

* specify the max number of parallel hdf sub-files:

OBPSF:maximum number of parallel

sub-files 2

compressed

open

mcih350.show

frequency to flush

5

exit

turn off twilight zone

implicit

* lian

\# outer box is done explicitly

choose grids for implicit

all=implicit

uppersheath=explicit

lowersheath=explicit

samplestream=explicit

done

pde parameters

passive scalar diffusion coefficient $5 \mathrm{e}-4$

fluid density

1.0

nu

1

*

* turn on second order artificial diffusion

* OBPDE:second-order artificial diffusion \$ad2
* OBPDE:ad21,ad22 \$ad21,\$ad22

* \#OBPDE:fourth-order artificial

diffusion 1

* \#OBPDE:ad41,ad42 1,1

done

final time 35

times to plot .01

*plot and always wait

\#

pressure solver options

choose best iterative solver

\# these tolerances are chosen for PETSc

relative tolerance

\$rtolp

absolute tolerance

\$atolp

debug

\$pdebug

exit

\#

implicit time step solver options

\$solver

\#choose best iterative solver

\# these tolerances are chosen for PETSc

relative tolerance

\$rtol

absolute tolerance

\$atol

debug

\$idebug

exit

\#

\#

debug \$debug

\#

boundary conditions

all=noSlipWall

uppersheath $(0,1)=$ inflowWithVelocityGi ven, uniform $(\mathrm{p}=1, \mathrm{v}=-.48)$

lowersheath $(1,1)=$ inflowWithVelocityGi ven, uniform $(\mathrm{p}=1, \mathrm{v}=.48)$

samplestream $(1,0)=$ outflow, uniform $(\mathrm{p}=0, \mathrm{u}=0)$ 


\author{
samplestream $(0,0)=$ inflowWithVelocity \\ Given, uniform $(\mathrm{p}=1 ., \mathrm{u}=.04)$ \\ done \\ initial conditions \\ uniform flow \\ $\mathrm{p}=1$. \\ exit
}

project initial conditions time stepping parameters... recompute dt every 10 steps close time stepping exit 


\section{APPENDIX VIII}

*Create a channel that has three inlet channels

create mappings

*Making of the upper sheath stream rectangle

set corners

0. -11.51

lines

3220

exit

stretch coordinates

transform which mapping?

square

stretch

specify stretching along axis $=0$

stretching type

hyperbolic tangent

hyperbolic tangent parameters .5 .0311 .03 .5

exit

exit

boundary conditions

1110

share

8867

mappingName

uppersheath

exit

delete a mapping

square

*making of the lower sheath stream

rectangle

set corners

$0 .-1-.50 .0$

lines

3220

exit

stretch coordinates

transform which mapping?

square

stretch

specify stretching along axis $=0$

stretching type hyperbolic tangent

hyperbolic tangent parameters .5 .0311 .03 .5

exit

exit

boundary conditions

1110

share

12121513

mappingName

lowersheath

exit

delete a mapping

square

*Making the sample stream

rectangle

set corners

$-1.57 .50 .1$

lines

35064

exit

stretch coordinates

transform which mapping?

square

stretch

specify stretching along axis $=1$

stretching type

hyperbolic tangent

hyperbolic tangent parameters .5 .0311 .03 .5

exit

exit

boundary conditions

1111

share

2017128

mappingName

samplestream

exit

delete a mapping

square

Annulus

lines 
7548

inner and outer radii

.05 .1

centre for annulus

$-1.00 .5$

boundary conditions

$-1-110$

mappingName

molecule1

exit

*

*

Annulus

lines

7548

inner and outer radii

.05 .1

centre for annulus

$-1.20 .5 \quad \rightarrow$ trouble: .6 .25

boundary conditions

$-1-110$

mappingName

molecule2

exit

*

* now look at the mappings view mappings

uppersheath

lowersheath

samplestream

molecule1

molecule2

colour boundaries by share value

*pause

erase and exit

exit

generate an overlapping grid
* put the nonconforming grid first to be a lower

* priority than the back-ground samplestream

uppersheath

lowersheath

molecule1

molecule2

done

change parameters

prevent hole cutting

uppersheath

samplestream

lowersheath

samplestream

samplestream

uppersheath

samplestream

lowersheath

done

ghost points

all

222222

exit

* display intermediate

* set debug parameter

* 31

compute overlap

*change parameters

*interpolation type

* explicit for all grids

*exit

exit

*

save an overlapping grid

mci.hdf

mci

exit 


\section{APPENDIX IX}

* specify the overlapping grid to use:

mci.hdf

* Specify the equations we solve:

incompressible Navier Stokes

exit

$*$

* Next specify the file to save the results in.

* This file can be viewed with

Overture/bin/plotStuff.

show file options

compressed

open

mci.show

frequency to flush

3

exit

* display parameters

turn off twilight zone

degree in space

1

*

turn on moving grids

* *detect collisions

specify grids to move

rigid body

density

1000

moments of inertia

.1

initial centre of mass

$-1.20 .5$

initial velocity

.22

done

molecule1

done

rigid body

density

1000

moments of inertia

.1

initial velocity

.21 initial centre of mass

$-1.20 .5$

done

molecule2

done

done

* choose implicit time stepping:

implicit

* but integrate the square explicitly:

choose grids for implicit

all=implicit

*uppersheath=explicit

*lowersheath=explicit

*samplestream=explicit

*molecule $1=$ explicit

*molecule2=explicit

done

*

final time 35

times to plot .001

plot and always wait

** no plotting

pde parameters

nu

1

* turn off second order artificial diffusion

* turn off fourth order artificial diffusion

* turn on second order artificial diffusion

*OBPDE:second-order artificial diffusion 1

*OBPDE: ad21, ad22 2,2

*OBPDE:fourth-order artificial diffusion 1

*OBPDE:ad41,ad42 1,1

done

*

* OBPSF:show variable: vorticity 1

* OBPSF:show variable: divergence 1

*

$* \mathrm{cfl}$

$* .25$

boundary conditions

all=noSlipWall 


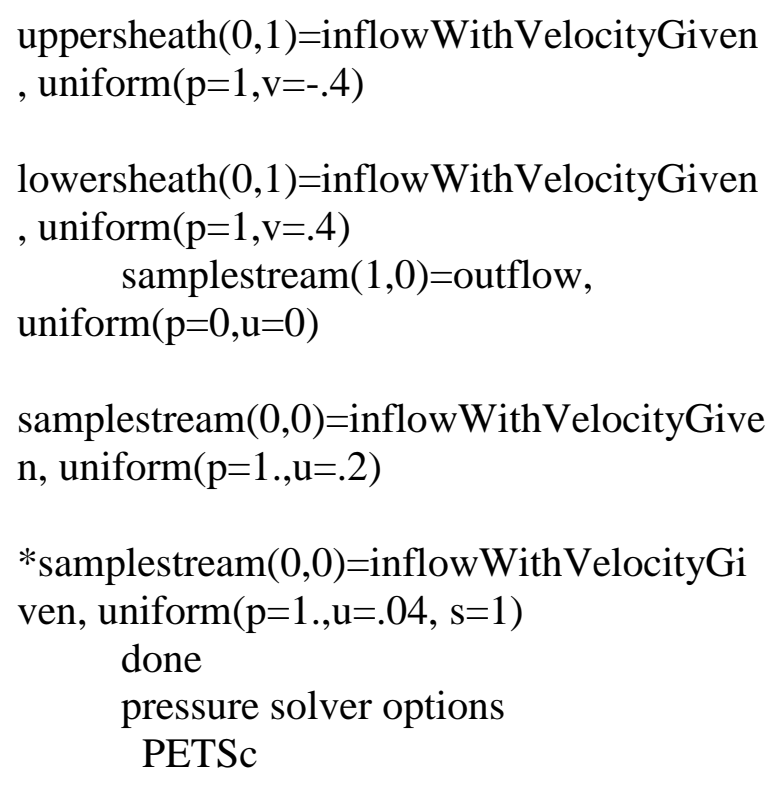

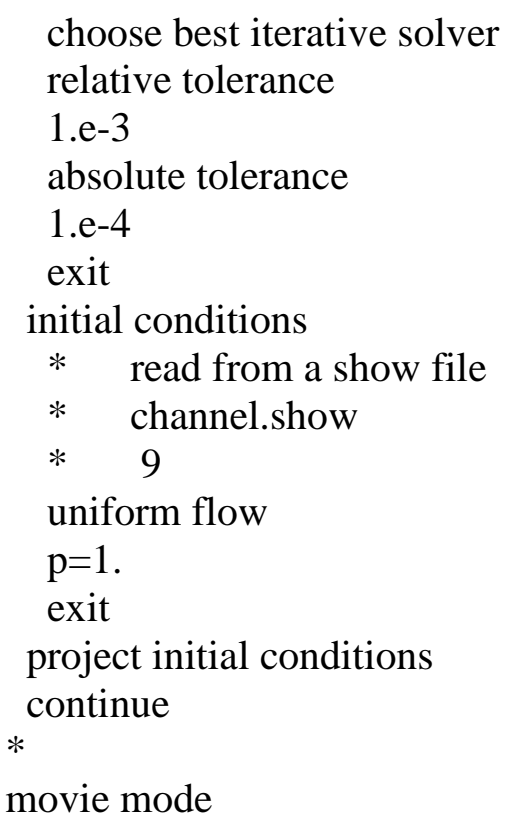




\section{APPENDIX X}

\# specify the overlapping grid to use:

mci.hdf

\# Specify the equations we solve: incompressible Navier Stokes

passive scalar advection

exit

\#

\$ts

SimplicitVariation

*

* lian showfile show file options

* specify the max number of parallel hdf sub-files:

OBPSF:maximum number of

parallel sub-files 2

compressed

open

mci.show

frequency to flush

20

exit

turn off twilight zone

implicit

* lian

\# outer box is done explicitly

choose grids for implicit

all=implicit

uppersheath=explicit

lowersheath=explicit

samplestream=explicit

done

pde parameters

passive scalar diffusion coefficient $5 \mathrm{e}$ -

4

fluid density

1.0

$\mathrm{nu}$

1

*

* turn on second order artificial

diffusion
* OBPDE:second-order artificial

diffusion $\$ a \mathrm{ad} 2$

* OBPDE:ad21,ad22 \$ad21,\$ad22

* \#OBPDE:fourth-order artificial

diffusion 1

* \#OBPDE:ad41,ad42 1,1

done

final time 10

times to plot .01

*plot and always wait

\#

pressure solver options

choose best iterative solver

\# these tolerances are chosen for

PETSc

relative tolerance

\$rtolp

absolute tolerance

\$atolp

debug

\$pdebug

exit

\#

implicit time step solver options

\$solver

\#choose best iterative solver

\# these tolerances are chosen for

PETSc

relative tolerance

\$rtol

absolute tolerance

\$atol

debug

\$idebug

exit

\#

\#

debug \$debug

\#

boundary conditions

all=noSlipWall 


$\begin{array}{lc} & \text { done } \\ \text { uppersheath }(0,1)=\text { inflowWithVelocityGi } & \text { initial conditions } \\ \text { ven, uniform }(\mathrm{p}=1, \mathrm{v}=-.48) & \text { uniform flow } \\ & \mathrm{p}=1 . \\ \text { lowersheath }(1,1)=\text { inflowWithVelocityGi } & \text { exit } \\ \text { ven, uniform }(\mathrm{p}=1, \mathrm{v}=.48) & \text { project initial conditions } \\ \quad \text { samplestream }(1,0)=\text { outflow, } & \text { time stepping parameters... } \\ \text { uniform }(\mathrm{p}=0, \mathrm{u}=0) & \text { recompute dt every } 10 \text { steps } \\ & \text { close time stepping } \\ \text { samplestream }(0,0)=\text { inflowWithVelocity } & \text { exit } \\ \text { Given, uniform }(\mathrm{p}=1 ., \mathrm{u}=.04, \mathrm{~s}=1) & \end{array}$




\section{APPENDIX XI}

*Create a 3-D channel

*share
$* 3,3,3,8,5,6$
mappingName
lowersheath
exit
view mappings
choose all
exit
exit
generate an overlapping grid
samplestream
uppersheath
lowersheath
done
change parameters
*interpolation type
$\quad *$ explicit for all grids
prevent hole cutting
lowersheath
samplestream
uppersheath
samplestream
samplestream
lowersheath
samplestream
uppersheath
done
ghost points
all
222222
exit
compute overlap
exit
save an overlapping grid
3 d.hdf
$3 \mathrm{~d}$
Exit
create mapping

box

set corners

$-1,8.50,1.0,1$.

lines

*x y z

1001010

boundary conditions

*left right bottom,top back,front

$1,1,1,1,1,1$

*share

*left,right bottom,top back, front

$* 1,2,3,4,5,6$

mappingName

samplestream

exit

*creating the uppersheath

box

set corners

0,1 . 1.,2 0,1 .

lines

$10,10,10$

boundary conditions

$1,1,0,1,1,1$

*share

$* 4,4$ 4,7 5,6

mappingName

uppersheath

exit

*creating the lowersheath

box

set corners

$0,1 .-1,0.0,1$.

lines

$10,10,10$

boundary conditions

$1,1,1,0,1,1$ 


\section{APPENDIX XII}

* specify the overlapping grid to use:

3d.hdf

* Specify the equations we solve: incompressible Navier Stokes

passive scalar advection

exit

*

* Next specify the file to save the results in.

* This file can be viewed with

Overture/bin/plotStuff.

show file options

compressed

open

3d.show

frequency to flush

1

exit

* display parameters

turn off twilight zone

*

* choose implicit time stepping:

implicit

* but integrate the square explicitly:

choose grids for implicit

all=implicit

uppersheath=explicit

lowersheath=explicit

samplestream=explicit

done

pde parameters

* kThermal \$kThermal passive scalar diffusion coefficient $5 \mathrm{e}-4$ fluid density

1.0

done

*

final time 35

times to plot .05

plot and always wait

** no plotting

pde parameters

nu

1
* turn off second order artificial diffusion

* turn off fourth order artificial diffusion

* turn on second order artificial diffusion

* OBPDE:second-order artificial

diffusion 1

* OBPDE:ad21, ad22 2,2

* OBPDE:fourth-order artificial

diffusion 1

* OBPDE:ad41,ad42 1,1

done

*

* OBPSF:show variable: vorticity 1

* OBPSF:show variable: divergence 1

*

$* \mathrm{cfl}$

* .25

boundary conditions

all=noSlipWall

uppersheath $(1,1)=$ inflowWithVelocityGiven

, uniform $(\mathrm{p}=1, \mathrm{v}=-.48)$

lowersheath $(0,1)=$ inflowWithVelocityGiven

, uniform $(\mathrm{p}=1, \mathrm{v}=.48)$

samplestream $(1,0)=$ outflow,

uniform $(\mathrm{p}=0, \mathrm{u}=0)$

samplestream $(0,0)=$ inflowWithVelocityGive

$\mathrm{n}, \operatorname{uniform}(\mathrm{p}=1 ., \mathrm{u}=.04, \mathrm{~s}=1)$

done

pressure solver options

* PETSc

choose best iterative solver

relative tolerance

1.e-3

absolute tolerance

1.e-4

exit

initial conditions

* read from a show file

* channel.show

* 9

uniform flow 
$\mathrm{p}=1$.

exit

project initial conditions

continue
*

movie mode

finish 


\section{REFERENCES}

Abcam. (1998). Introdution to flow cytometry. Retrieved 4 3, 2010, from http://www.abcam.com/index.html?pageconfig=resource\&rid=11446

Basmadjian, D. (2007). Mass Transfer and Separation Processes: Principles and Applications. CRC Press.

Bioscience Technology. (2009, 09). Retrieved 1 20, 2010, from

http://www.biosciencetechnology.com/uploadedImages/BST/Products/2009/09/bt0909mi lliporeneuro.jpg

Edwards, S. (2009). Cardiff School of Engineering. Retrieved 10 5, 2009, from http://www.cardiff.ac.uk/medic/aboutus/departments/haematology/cytometry/fluidics/ind ex.html

Graham, M. D. (2003). The Coulter Principle: Foundation of an Industry. JALA , 72-81.

Harnett, C. K. (2009, 6 29). Purpose of micro channel research. (K. A. Schmidt, Interviewer)

Henshaw. (1998). Overture. Retrieved 8 17, 2009, from Overture:

https://computation.llnl.gov/casc/Overture/tech_brochure/Overture_01.pdf

Henshaw. (2004, December). Short Course: Solving PDE's on Overlapping Grids with the Overture Framework. Livermore, CA.

Henshaw. (2008). Solving Partial Differential Equation on Overlapping Grids. Lawrence Livermore National Laboratory.

Henshaw, W. D. (2006, 9 29). Cgins: A Solver for the Incompressible Navier-Stokes Equations Refernce Manual. Livermore, CA. Retrieved from Overture.

Hexley, P., \& Elliott, J. (2010, 3 30). Flow Cytometry Core Facility. Retrieved 4 3, 2010, from Flow Cytometry Core Facility: http://flow.csc.mrc.ac.uk/?page_id=302

Hoffmann, K. (1989). Computational FLuid Dynamics for Engineers. Austin: Engineering Education System. 
Houwen, B. (2003, 11). Health Care Industry. Retrieved 4 18, 2010, from Fifty years of hematology innovation: the Coulter Principle - Retrospective:

http://findarticles.com/p/articles/mi_m3230/is_11_35/ai_111351102/

J.H., N., F., K., J., B., P.M., S., \& M.J., V. (2004). Integrated Coulter counter based on 2dimensional liquid aperture control. Sensors and Actuators , 44-50.

Kyle Chand, W. H. (2007, 10). Overture Demo Introduction. Retrieved 8 17, 2009, from Overture:

https://computation.llnl.gov/casc/Overture/henshaw/overtureDocumentation/overtureDoc umentation.html

Lee, G.-B., Chang, C.-C., Huang, S.-B., \& Yang, R.-J. (2005). The hydrodynamic focusing effect inside rectangular microchannels. Journal of Micromechanics and Microengineering , 1024-1031.

Mills, A. (2001). Mass Transfer. Upper Saddle River: Prentice Hall.

Okiishi, M. Y. (2006). Fundamentals of Fluid Mechanics. Hoboken: John Wiley \& Sons.

Scott, R., Sethu, P., \& Harnett, C. (2008). Three-dimensional hydrodynamic focusing in a microfluidic Coulter counter. Review of Scientific Instruments , 3 .

Shuler, L. M., Aris, R., \& Tsuchiya, H. M. (1972). Hydrodynamic Focusing and Electronic Cell-Sizing Techniques. Applied Microbiology, 384-388.

The Coutler Particle Counter/Sizer. (n.d.). Retrieved 11 29, 2009, from www.scienceprojects.com/Coulter/Coulter.htm

Walsh, P., Walsh, E., \& Davies, M. (2007). On the out-of-plane divergence of streamtubes in planar mini-scale flow focusing devices. International Journal of Heat and Flow , 44-53.

Wu, Z., \& Nguyen, N.-T. (2005). Hydrodynamic focusing in microchannels under consideration of diffusive despersion: theories and experiments. Sensors and Actuators $B$ $107,965-974$. 


$\begin{array}{lr} & \\ & 5406 \text { Sunnybrook Dr. } \\ \text { Louisville, KY } 40214 \\ (812) 430-7373 \\ \text { kyle A. Schmidt } \\ \text { kaschm02@gmail.com }\end{array}$

\section{Objective}

Full time mechanical engineering position using hard work to apply engineering, communication, and time management skills.

\section{Education}

Master of Engineering Degree in Mechanical Engineering

Expected May 2010

J.B. Speed School of Engineering, University of Louisville, KY

G.P.A 3.3/4.0

Bachelor of Science Degree in Mechanical Engineering

May 2009

J.B. Speed School of Engineering, University of Louisville, KY

G.P.A 3.0/4.0

\section{Professional Experience}

Samtec Inc.

3 Engineering Co-op terms, 2 part-time terms

New Albany, IN

- Created AutoCAD and SolidWorks drawings of electronic connectors

- Updated AutoCAD and SolidWorks prints after engineering changes

- Applied machine design, tooling, injection molding, and engineering design to create and debug multiple high speed electronic connectors

- Designed right angle supports, flex connectors, and tooling to separate mated connectors

\section{Schott Home Tech}

Part time Engineering Intern

Louisville, $\mathrm{KY}$

- Displayed gasket capabilities on sample glass for consumer product realization

- Created tooling to improve gasket molding process for borders of sample glass

- Completed regulation standard on kitchen appliances to insure product quality

University of Louisville

Research Assistant in CFD Lab

Louisville, $\mathrm{KY}$

- Generated computerized micro-channel utilizing Overture software

- Performed CFD analysis on micro-channel

- Analyzed numerical results of CFD to find diffusion, velocity, and temperature profiles

\section{Applied Experience}

CFD Analysis Lab: Applied knowledge of heat transfer, mass transfer, and fluid dynamics to optimize particle flow through micro-channels. Researched 2-D and 3-D micro-channel grid sensitivities to validate computer generated numerical results.

\section{Employment Experience}

Valvoline

Certified Technician

- Changed oil on the bottom side of the pit

- Maintained the cleanliness of the pit

- Satisfied customer service expectation by providing them with quick service and care of their automotive vehicles

\section{Skills Summary}

$\begin{array}{lll}\text { - SolidWorks } & - \text { Overture } & - \text { Data Acquisition } \\ \text { - SolidEdge } & - \text { LabVIEW } & - \text { MatLAB } \\ \text { - ANSYS Introduction } & - \text { AutoCAD } & \end{array}$

- Gained welding, painting, and grinding experience through reconditioning classic cars

\section{Activities and Awards}

- EPSCoR Fellowship

- Worked 15-20 hours per week while being a full time student

- Used imagination, creativity, and patience to restore and rebuild small block Chevrolet motors and classic cars

- Enjoy playing intramural sports

\section{References}

Provided upon request 